\title{
Lattice gas model in random medium and open boundaries: hydrodynamic and relaxation to the steady state. *
}

\author{
Mustapha Mourragui $^{1}$ and Enza Orlandi ${ }^{2}$
}

\begin{abstract}
We consider a lattice gas interacting by the exclusion rule in the presence of a random field given by i.i.d. bounded random variables in a bounded domain in contact with particles reservoir at different densities. We show, in dimensions $d \geq 3$, that the rescaled empirical density field almost surely, with respect to the random field, converges to the unique weak solution of a non linear parabolic equation having the diffusion matrix determined by the statistical properties of the external random field and boundary conditions determined by the density of the reservoir. Further we show that the rescaled empirical density field, in the stationary regime, almost surely with respect to the random field, converges to the solution of the associated stationary transport equation.
\end{abstract}

\section{Introduction}

In the last years there has been several papers devoted in understanding macroscopic properties of non equilibrium systems. Typical examples are systems in contact with two thermostats at different temperature or with two reservoirs at different densities. A mathematical model of open systems is provided by stochastic models of interacting particles systems performing a local reversible dynamics (for example a reversible hopping dynamics) in a domain and some external mechanism of creation and annihilation of particles on the boundary of the domain, modeling the reservoirs, which makes the full process non reversible. The first question that one might ask for these systems is the derivation of the hydrodynamic behavior (law of large number) for the locally conserved field in the non stationary and stationary regime. There has been important classes of models, see for example [ELS1,2], [DFIP], [KLO] in which it has been proved the law of large numbers for the empirical density in the stationary regime. Typical generic feature of these systems is that they exhibit long range correlation in their steady state. These long range correlations have been calculated from the microscopic dynamics only in very few cases; mainly in the case of the symmetric exclusion process, see [Sp1], the asymmetric exclusion process, see [DEL], and in the weakly asymmetric exclusion process, see [DELO]. More recently breakthroughs were achieved analyzing the large deviations principle for the stationary measure. We refer to [BSGJL] for a review of works on the statistical mechanics of non equilibrium processes based on the analysis of large deviations properties of microscopic systems.

In this paper we focus on the first step. We derive the macroscopic limit in the stationary and not stationary regime (hydrodynamic limit) for a particles system evolving according to local- conservative dynamics (Kawasaki) with hard core exclusion rule and with rates depending on a quenched random field

* work supported by INDAM-CNRS, Roma TRE, University of Rouen.

1 Université de Rouen, LMRS, UMR 6085, Avenue de l'Université, BP. 12, 76801, Saint Etienne du Rouvray, France. Mustapha.Mourragui@univ-rouen.fr

2 Dipartimento di Matematica, Universitá di Roma Tre, L.go S.Murialdo 1, 00146 Roma, Italy. orlandi@mat.uniroma3.it Key Words : Random environment, Nongradient systems, Stationary nonequilibrium states. 2000 Mathematics Subject Classification. Primary 82C22, 60K35, Secondary 60F10, 82C35. 
in a cylinder domain $d \geq 3$ in which the basis, denoted $\Gamma$, are kept at different densities. The restriction on the dimensions is only technical. We comment on this later. The rates are chosen so that the system satisfies a detailed balance condition with respect to a family of random Bernoulli measures (the random field Ising model at infinite temperature). To model the presence of the reservoirs, as in previous papers, we superimpose at the boundary, to the local-conservative dynamics, a jump dynamics (creation and destruction of particle). The rates of the birth and death process depend on the realizations of the random field and are chosen so that a random Bernoulli measure with a suitable choice of the chemical potential is reversible for it. This latter dynamic is of course not conservative and keeps the fixed value of the density on the boundary. There is a flow of density through the full system and the full dynamic is not reversible. The bulk dynamic models electron transport in doped crystals. In this case the exclusion rule is given by the Pauli principle and the presence of impurities in the crystals is the origin of the presence of quenched random field, see $[\mathrm{KW}]$. The presence of the random field together with the exclusion rule makes the problem high not trivial. The transport properties of such systems in the case of periodic boundary condition on $\Gamma$ has been studied by Faggionato and Martinelli, [FM]. They derived in $d \geq 3$, the hydrodynamic limit and gave a variational formula for the bulk diffusion, equivalent to the Green-Kubo formula. They proved that the bulk diffusion is a deterministic quantity depending on the statistical properties of the random field. Later, Quastel [Q] derived in all dimensions for the same model investigated by $[\mathrm{FM}]$ the hydrodynamic limit for the local empirical density. Applying the method proposed by Quastel, we could extend our results in all dimensions. Since our aim is to understand the role of the randomness in the non stationary and stationary state and not the role of dimensions in the bulk dynamics we state and prove our results in $d \geq 3$. Dynamical Large deviations for the same model and always with periodic boundary conditions have been derived in [MO] as special case of a more general system discussed there. The bulk dynamics is of the so-called nongradient type. Roughly speaking, the gradient condition says that the microscopic current is already the gradient of a function of the density field. Further it is not translation invariant, for a given disorder configuration. In order to prove the hydrodynamic behavior of the system, we follow the entropy method introduced by Guo, Papanicolaou and Varadhan [GPV]. It relies on an estimate of the entropy of the states of process with respect to a reference invariant state. By the general theory of Markov Processes the entropy of the state of a process with respect to an invariant state decreases in time. The main problem is that in the model considered the reference invariant state is not explicitly known. To overcome this difficulty we compute the entropy of the state of the process with respect to a product measure with slowly varying profile. Since this measure is not invariant, the entropy does not need to decrease and we need to estimate the rate at which it increases. This type of strategy has been used in previous papers dealing with the same type of problems, see [KLO] and [LMS], which considered generalized exclusion process of non gradient type. The main difference with the previous mentioned papers is the presence of the randomness in the model considered here. This forces to take on the boundary a jump process depending on the external random field. Important step to derive the final results is then a convenient application of the ergodic theorem. Further we show that the empirical density field obeys a law of large numbers with respect to the stationary random measures (hydrostatic). This is achieved proving that it is possible to derive the hydrodynamic for the evolution of the empirical measures starting from any initial particle configurations distributed according to the stationary measure, even though it is not possible to identify the profile. Then we exploit that the stationary solution of 
the parabolic nonlinear equation is unique and is a global attractor for the evolution. These two ingredients allow to conclude. Similar strategy for proving the hydrostatic is used in the paper in preparation by Farfan Vargas, Landim and Mourragui, [FLM].

\section{The model and the main results}

\subsection{The model}

We consider the $d$ - dimensional lattice $\mathbb{Z}^{d}$ with sites $x=\left(x_{1}, \ldots, x_{d}\right)$ and canonical basis $\mathcal{E}=\left\{e_{1}, \ldots, e_{d}\right\}$ and we assume in all the paper that $d \geq 3$. We denote by $\Lambda:=[-1,1] \times \mathbb{T}^{d-1}$, where $\mathbb{T}^{d-1}$ is the $(d-1)$ dimensional torus of diameter 1 and by $\Gamma$ the boundary of $\Lambda$.

Fix an integer $N \geq 1$. Denote by $\Lambda_{N} \equiv\{-N, \cdots, N\} \times \mathbb{T}_{N}^{d-1}$ the cylinder in $\mathbb{Z}^{d}$ of length $2 N+1$ with basis the $(d-1)$-dimensional discrete torus $\mathbb{T}_{N}^{d-1}$ and by $\Gamma_{N}=\left\{x \in \Lambda_{N} \mid x_{1}= \pm N\right\}$ the boundary of $\Lambda_{N}$. The elements of $\Lambda_{N}$ will be denoted by letters $x, y, \ldots$ and the elements of $\Lambda$ by $u, v, \ldots$

For a fixed $A>0$, let $\Sigma_{D}=[-A, A]^{\mathbb{Z}^{d}}$ be the set of disorder configurations on $\mathbb{Z}^{d}$. On $\Sigma_{D}$ we define a product, translation invariant probability measure $\mathbb{P}$. We denote by $\mathbb{E}$ the expectation with respect to $\mathbb{P}$, and by $\alpha \equiv\left\{\alpha(x), x \in \mathbb{Z}^{d}\right\}, \alpha(x) \in[-A, A]$, a disorder configuration in $\Sigma_{D}$. A configuration $\alpha \in \Sigma_{D}$ induces in a natural way a disorder configuration $\alpha_{N}$ on $\Lambda_{N}$, by identifying a cube centered at the origin of side $2 N+1$ with $\Lambda_{N}$. By a slight abuse of notation whenever in the following we refer to a disorder configuration either on $\Lambda_{N}$ or on $\mathbb{Z}^{d}$ we denote it by $\alpha$. We denote by $\mathcal{S}_{N} \equiv\{0,1\}^{\Lambda_{N}}$ and $\mathcal{S} \equiv\{0,1\}^{\mathbb{Z}^{d}}$ the configuration spaces, both equipped with the product topology; elements of $\mathcal{S}_{N}$ or $\mathcal{S}$ are denoted by $\eta$, so that $\eta(x)=1$, resp 0 , if the site $x$ is occupied, resp empty, for the configuration $\eta$. Given $\alpha \in \Sigma_{D}$, we consider the random Hamiltonian $H^{\alpha}: \mathcal{S}_{N} \rightarrow \mathbb{R}$,

$$
H^{\alpha}(\eta)=-\sum_{x \in \Lambda_{N}} \alpha(x) \eta(x)
$$

We denote by $\mu_{N}^{\alpha, \lambda}$ the grand canonical random Gibbs measure on $\mathcal{S}_{N}$ associated to the Hamiltonian (2.1) with chemical potential $\lambda \in \mathbb{R}$, i.e the random Bernoulli product measure

$$
\mu_{N}^{\alpha, \lambda}(\eta)=\prod_{x \in \Lambda_{N}}\left\{\frac{e^{[\alpha(x)+\lambda] \eta(x)}}{e^{[\alpha(x)+\lambda]}+1}\right\}
$$

When $\lambda=0$, we simply write $\mu_{N}^{\alpha}$. We denote by $\mu^{\alpha, \lambda}(\cdot)$ and when $\lambda=0, \mu^{\alpha}(\cdot)$ the measure (2.2) on the infinite product space $\mathcal{S}$. Moreover, for a probability measure $\mu$ and a bounded function $f$, both defined on $\mathcal{S}$ or $\mathcal{S}_{N}$, we denote by $\mathbf{E}^{\mu}(f)$ the expectation of $f$ with respect to $\mu$. We need to introduce also the canonical measures $\nu_{\rho}^{\alpha, N}$,

$$
\nu_{\rho}^{\alpha, N}(\cdot)=\mu_{N}^{\alpha, \lambda}\left(\cdot\left|\sum_{x \in \Lambda_{N}} \eta_{x}=\rho\right| \Lambda_{N} \mid\right)
$$

for $\rho \in\left[0, \frac{1}{\left|\Lambda_{N}\right|}, \ldots, 1\right]$. It is well known $[\mathrm{CM}]$ that the canonical and the grand canonical measures are closely related if the chemical potential $\lambda$ is chosen canonical conjugate to the density $\rho$, in the sense that 
the average density with respect to $\mu_{N}^{\alpha, \lambda}$ is equal to $\rho$. So as in $[\mathrm{FM}]$ one can define the random empirical chemical potential and the annealed chemical potential $\lambda_{0}(\rho)$. To our aim it is enough to consider $\lambda_{0}(\rho)$. For $\rho \in[0,1]$, the function $\lambda_{0}(\rho)$ is defined as the unique $\lambda$ so that

$$
\mathbb{E}\left[\int \eta(0) d \mu^{\alpha, \lambda}(\eta)\right]=\mathbb{E}\left[\frac{e^{\alpha(0)+\lambda}}{1+e^{\alpha(0)+\lambda}}\right]=\rho .
$$

We will consider as reference measure the random Bernoulli product measure $\nu_{\rho(\cdot)}^{\alpha, N}$ on $\mathcal{S}_{N}$ defined for positive profile $\rho: \Lambda \rightarrow(0,1)$ by

$$
\nu_{\rho(\cdot)}^{\alpha, N}(\eta)=\prod_{x \in \Lambda_{N}}\left\{\frac{e^{\left[\alpha(x)+\lambda_{0}(\rho(x / N))\right] \eta(x)}}{e^{\left[\alpha(x)+\lambda_{0}(\rho(x / N))\right]}+1}\right\}
$$

if $\rho(\cdot) \equiv \rho$ is constant, we shall denote simply $\nu_{\rho(\cdot)}^{\alpha, N}=\nu_{\rho}^{\alpha, N}$. We denote by $\eta^{x, y}$ the configuration obtained from $\eta$ by interchanging the values at $x$ and $y$ :

$$
\eta^{x, y}(z)=\left\{\begin{array}{llr}
\eta(x) & \text { if } & z=y \\
\eta(y) & \text { if } & z=x \\
\eta(z) & \text { otherwise }
\end{array}\right.
$$

and by $\eta^{x}$ the configuration obtained from $\eta$ by flipping the occupation number at site $x$ :

$$
\eta^{x}(z)=\left\{\begin{array}{l}
\eta(z) \quad \text { if } \quad z \neq x \\
1-\eta(x) \quad \text { if } \quad z=x .
\end{array}\right.
$$

Further, for $f: \mathcal{S}_{N} \rightarrow \mathbb{R}, x, y \in \Lambda_{N}$, we denote

$$
\left(\nabla_{x, y} f\right)(\eta)=f\left(\eta^{x, y}\right)-f(\eta)
$$

The disordered exclusion process on $\Lambda_{N}$ with random reservoirs at its boundary $\Gamma_{N}$ is the Markov process on $\mathcal{S}_{N}$ whose generator $\mathcal{L}_{N}$ can be decomposed as

$$
\mathcal{L}_{N}=\mathcal{L}_{N}^{0}+\mathcal{L}_{N}^{b}
$$

where the generators $\mathcal{L}_{N}^{0}, \mathcal{L}_{N}^{b}$ act on function $f: \mathcal{S}_{N} \rightarrow \mathbb{R}$ as

$$
\left(\mathcal{L}_{N}^{0} f\right)(\eta)=\sum_{e \in \mathcal{E}} \sum_{x \in \Lambda_{N}, x+e \in \Lambda_{N}} C_{N}(x, x+e ; \eta)\left[\left(\nabla_{x, x+e} f\right)(\eta)\right]
$$

where $e$ is a generic element of $\mathcal{E}$, the rate $C_{N}$ is given by

$$
C_{N}(x, y ; \eta) \equiv C_{N}^{\alpha}(x, y ; \eta)=\exp \left\{-\frac{\left(\nabla_{x, y} H^{\alpha}\right)(\eta)}{2}\right\}
$$

and

$$
\left(\mathcal{L}_{N}^{b} f\right)(\eta)=\sum_{x \in \Gamma_{N}} C^{b}(x / N, \eta)\left[f\left(\eta^{x}\right)-f(\eta)\right]
$$


To define the rate $C^{b}(x / N, \eta)$ we fix a function $b(\cdot)$ on $\Gamma$, representing the density of the reservoirs. We assume that $b(\cdot)$ is the restriction on $\Gamma$ of a smooth function $\gamma(\cdot)$ defined on a neighborhood $V$ of $\Lambda, \gamma: V \rightarrow(0,1)$ and $\gamma(u)=b(u)$ for $u \in \Gamma$. The rate $C^{b}$ is chosen so that $\mathcal{L}_{N}^{b}$ is reversible with respect to $\nu_{\gamma(\cdot)}^{\alpha, N}$

$$
C^{b}(x / N, \eta)=\eta(x) \exp \left\{-\frac{\alpha(x)+\lambda_{0}\left(b\left(\frac{x}{N}\right)\right)}{2}\right\}+(1-\eta(x)) \exp \left\{\frac{\alpha(x)+\lambda_{0}\left(b\left(\frac{x}{N}\right)\right)}{2}\right\} .
$$

The first term in (2.11) is the creation rate, the second one is the annihilation rate. Next we recall the relevant properties of $C_{N}(x, y ; \eta)$ :

a) detailed balance condition with respect to the measure (2.2),

b) positivity and boundedness: there exists $a>0$ such that

$$
a^{-1} \leq C_{N}(x, y ; \eta) \leq a,
$$

c) translation covariant:

$$
C_{N}^{\alpha}(x, y ; \eta)=C_{N}^{\tau_{z} \alpha}\left(x-z, y-z ; \tau_{z} \eta\right)=\tau_{z} C_{N}^{\alpha}(x-z, y-z ; \eta)
$$

where for $z$ in $\mathbb{Z}^{d}, \tau_{z}$ denotes the space shift by $z$ units on $\mathcal{S} \times \Sigma_{D}$ defined for all $\eta \in \mathcal{S}, \alpha \in \Sigma_{D}$ and $g: \mathcal{S} \times \Sigma_{D} \rightarrow \mathbb{R}$ by

$$
\left(\tau_{z} \eta\right)(x)=\eta(x+z),\left(\tau_{z} \alpha\right)(x)=\alpha(x+z),\left(\tau_{z} g\right)(\eta, \alpha)=g\left(\tau_{z} \eta, \tau_{z} \alpha\right) .
$$

We omit to write in the notation the explicit dependence on the randomness $\alpha$, unless there is an ambiguity. The process arising from the full generator (2.7) is then a superposition of a dynamics with a conservation law (the Kawasaki random dynamics) acting on the whole $\Lambda_{N}$ and a birth and death process acting on $\Gamma$. Remark that if $b(\cdot) \equiv b_{0}$ for some positive constant $b_{0}$, then the generator $\mathcal{L}_{N}$, see $(2.7)$, is self-adjoint in $L^{2}\left(\nu_{b_{0}}^{\alpha, N}\right)$ and the measure $\nu_{b_{0}}^{\alpha, N}$ is the stationary measure for the full dynamics $\mathcal{L}_{N}$. In the general case, when $b(\cdot)$ is not constant, since the Markov process on $\mathcal{S}_{N}$ with generator (2.7), is irreducible for all $N \geq 1$, there exists always an unique invariant measure but in general cannot be written in an explicit form.

\subsection{The macroscopic equation}

The macroscopic evolution of the local particles density $\rho$ is described by the quasi linear parabolic equation

$$
\left\{\begin{array}{l}
\partial_{t} \rho=\nabla \cdot(D(\rho) \nabla \rho), \\
\rho(0, \cdot)=\rho_{0}, \\
\left.\rho(t, \cdot)\right|_{\Gamma}=b(\cdot) \quad \text { for } t>0,
\end{array}\right.
$$

where $D(\rho)$ is the diffusion matrix given in $(2.17), b(\cdot) \in C^{1}(\Gamma)$ represents the interaction with the reservoirs appearing as boundary conditions to be imposed on the solution, see its definition before $(2.11)$, and $\rho_{0}$ : $\Lambda \rightarrow[0,1]$ is the initial profile. The diffusion matrix is the one derived in [FM]. To define it, let *

$$
\mathbb{G} \equiv\left\{g: \mathcal{S} \times \Lambda_{D} \rightarrow \mathbb{R} ; \text { local and bounded }\right\}
$$

* A function $g: \mathcal{S} \times \Lambda_{D} \rightarrow I R$ is local if the support of $g, \Delta_{g}$, i.e. the smallest subset of $Z^{d}$ such that $g$ depends only on $\left\{(\eta(x), \alpha(x)) x \in \Delta_{g}\right\}$, is finite. The function $g$ is bounded if $\sup _{\eta} \sup _{\alpha}|g(\eta, \alpha)|<\infty$.

$29 /$ october $/ 2018 ; 13: 48$ 
and for $g \in \mathbb{G}, \Gamma_{g}(\eta)=\sum_{x \in \mathbb{Z}^{d}}\left(\tau_{x} g\right)(\eta, \alpha)$. The $\Gamma_{g}(\eta)$ is a formal expression, but the difference $\nabla_{0, e} \Gamma_{g}(\eta)=$ $\Gamma_{g}\left(\eta^{0, e}\right)-\Gamma_{g}(\eta)$ for $e \in \mathcal{E}$ is meaningful. For each $\rho \in(0,1)$, let $D(\rho)=\left\{D_{i, j}(\rho), 1 \leq i, j \leq d\right\}$ be the symmetric matrix defined, for every $a \in \mathbb{R}^{d}$, by the variational formula

$$
(a \cdot D(\rho) a)=\frac{1}{2 \chi(\rho)} \inf _{g \in G_{i}} \sum_{i=1}^{d} \mathbb{E}\left[\mathbf{E}^{\mu^{\alpha, \lambda_{0}(\rho)}}\left(C^{0}\left(0, e_{i} ; \eta\right)\left\{a_{i} \nabla_{0, e_{i}} \eta(0)+\left(\nabla_{0, e_{i}} \Gamma_{g}\right)(\eta)\right\}^{2}\right)\right]
$$

where $\lambda_{0}(\rho)$ is defined in $(2.3), \chi(\rho)$ is the static compressibility given by

$$
\chi(\rho)=\mathbb{E}\left[\int \eta(0)^{2} d \mu^{\alpha, \lambda_{0}(\rho)}(\eta)-\left(\int \eta(0) d \mu^{\alpha, \lambda_{0}(\rho)}(\eta)\right)^{2}\right],
$$

for $a, b \in \mathbb{R}^{d},(a \cdot b)$ is the scalar vector product of $a$ and $b$ and, recall, $\mathbf{E}^{\mu^{\alpha, \lambda_{0}(\rho)}}(\cdot)$ is the expectation with respect to $\mu^{\alpha, \lambda_{0}(\rho)}$, see after $(2.2)$, the random Bernoulli product measure on $\mathcal{S}$ with annealed chemical potential $\lambda_{0}(\rho)$. In Theorem 2.1 of [FM] it has been proved, for $d \geq 3$ and for $\rho \in(0,1)$, the existence of the symmetric diffusion matrix defined in (2.17). Further it has been proved that the coefficients $D_{i, j}(\cdot)$ are nonlinear continuous functions in the open interval $(0,1)$ and there exists a constant $C>1$, depending on dimensions and bound on the random field, such that

$$
\frac{\mathbb{I}}{C} \leq D(\rho) \leq C \mathbb{I} \quad \rho \in(0,1)
$$

where $\mathbb{I}$ is the $d \times d$ identity matrix. One expects the matrix $D(\cdot)$ to be extended continuously to the closed interval $[0,1]$ and actually to be a smooth function of $\rho,[\mathrm{KW}]$. We will assume all trough the paper that $D(\cdot)$ is well defined in $[0,1]$ and Lipschitz in the open interval. The diffusion matrix $D(\rho)$ in a solid, in a regime of linear response, is linked to the mobility $\frac{1}{2} \sigma(\rho)$, see [Sp], via the Einstein relation

$$
D(\rho)=\frac{1}{2} \sigma(\rho) \chi(\rho)^{-1}
$$

The $\chi(\rho)$ is a smooth function of $\rho$ in $[0,1]$ and it can be easily proven from $(2.18)$ that

$$
\frac{1}{2} \rho(1-\rho) \leq \chi(\rho) \leq \rho(1-\rho) ; \quad \frac{1}{C} \rho(1-\rho) \mathbb{I} \leq \sigma(\rho) \leq C \mathbb{I} \rho(1-\rho),
$$

where $C$ is a constant that may change from one occurrence to the next.

Weak solutions By weak solution of $(2.15)$ we mean a function $\rho(\cdot, \cdot):[0, T] \times \Lambda \rightarrow \mathbb{R}$ satisfying (IB1) $\rho \in L^{2}\left((0, T) ; H^{1}(\Lambda)\right)$ :

$$
\int_{0}^{T} d s\left(\int_{\Lambda}\|\nabla \rho(s, u)\|^{2} d u\right)<\infty
$$

(IB2) For every function $G(t, u)=G_{t}(u)$ in $\mathcal{C}_{c}^{1,2}([0, T] \times \AA)$, where $\left.\AA_{=}=\right]-1,1\left[\times \mathbb{T}^{d-1}\right.$ and $\mathcal{C}_{c}^{1,2}([0, T] \times \AA)$ is the space of functions from $[0, T] \times \Lambda$ to $\mathbb{R}$ twice continuously differentiable in $\Lambda$ with continuous time derivative and having compact support in $\AA_{\text {we have }}$ 


$$
\begin{gathered}
\int_{\Lambda} d u\left\{G_{T}(u) \rho(T, u)-G_{0}(u) \rho(0, u)\right\}-\int_{0}^{T} d s \int_{\Lambda} d u\left(\partial_{s} G_{s}\right)(u) \rho(s, u) \\
=-\int_{0}^{T} d s\left\{\int_{\Lambda} d u D(\rho(s, u)) \nabla \rho(s, u) \cdot \nabla G_{s}(u)\right\}
\end{gathered}
$$

(IB3) For any $t \in(0, T], \operatorname{Tr}(\rho(t, \cdot))=b(\cdot)$, a.e..

(IB4) $\rho(0, u)=\rho_{0}(u)$. a.e.

Notice that, since the original particle model cannot have more than one particle at a lattice site any solution $\rho$ of $(2.15)$ is bounded between 0 and 1. The existence and uniqueness of the weak solution of (2.15) when $(2.19)$ holds and $D(\cdot)$ is Lipschitz continuous for $\rho \in(0,1)$, can be done using standard analysis tools. We refer to [LSU], chapter V or [DL]. Further, one immediately obtains by the characterization of $H^{-1}(\Lambda)$, see for example [E], page 283 , that $\partial_{t} \rho \in L^{2}\left(0, T ; H^{-1}(\Lambda)\right)$. Recall that $H^{-1}(\Lambda)$ is the dual of $H_{0}^{1}(\Lambda)$, i.e. the Banach space equipped with the norm

$$
\|v\|_{-1}=\sup _{f}\left\{\langle v, f\rangle:\|f\|_{H_{0}^{1}(\Lambda)} \leq 1\right\}
$$

Stationary solution We denote by $\bar{\rho}$ the stationary solution of $(2.15)$, i.e. a function from $\Lambda \rightarrow[0,1]$ so that $\bar{\rho} \in H^{1}(\Lambda)$, for $G \in \mathcal{C}_{c}^{2}(\Lambda)$ we have

$$
\left\{\begin{array}{l}
\int_{\Lambda} d u D(\bar{\rho}(u)) \nabla \bar{\rho}(u) \cdot \nabla G(u)=0 \\
\operatorname{Tr}(\bar{\rho}(\cdot))=b(\cdot), \text { a.e. }
\end{array}\right.
$$

\subsection{The main results}

For any $T>0$, we denote by $\left(\eta_{t}\right)_{t \in[0, T]}$ the Markov process on $\mathcal{S}_{N}$ with generator $N^{2} \mathcal{L}_{N}$ starting from $\eta_{0}=\eta$ and by $\mathbf{P}_{\eta}:=\mathbf{P}_{\eta}^{\alpha}$ its distribution when the initial configuration is $\eta$. We remind that we omit to write explicitly the dependence on $\alpha$. The $\mathbf{P}_{\eta}$ is a probability measure on the path space $D\left([0, T], \mathcal{S}_{N}\right)$, which we consider endowed with the Skorohod topology and the corresponding Borel $\sigma$-algebra. Expectation with respect to $\mathbf{P}_{\eta}$ is denoted by $\mathbf{E}_{\eta}$. If $\mu^{N}$ is a probability measure on $\mathcal{S}_{N}$ we denote $\mathbf{P}_{\mu^{N}}(\cdot)=\int_{\mathcal{S}_{N}} \mathbf{P}_{\eta}(\cdot) \mu^{N}(d \eta)$ and by $\mathbf{E}_{\mu^{N}}$ the expectation with respect to $\mathbf{P}_{\mu^{N}}$. For $t \in[0, T], \eta \in \mathcal{S}_{N}$, let the empirical measure $\pi_{t}^{N}$ be defined by

$$
\pi_{t}^{N}(\eta) \equiv \pi^{N}\left(d u ; \eta_{t}\right)=\frac{1}{N^{d}} \sum_{x \in \Lambda_{N}} \eta_{t}(x) \delta_{x / N}(d u)
$$

where $\delta_{u}(\cdot)$ is the Dirac measure on $\Lambda$ concentrated on $u$. Since $\eta(x) \in\{0,1\}$, relation (2.25) induces from $\mathbf{P}_{\mu^{N}}$ a distribution $Q_{\mu^{N}}$ on the Skorohod space $D\left([0, T], \mathcal{M}_{1}(\Lambda)\right)$, where $\mathcal{M}_{1}(\Lambda)$ is the set of positive Borel measures on $\Lambda$ with total mass bounded by 1 , endowed with the weak topology. Denote by $\mathcal{M}_{1}^{0}(\Lambda)$ the subset of $\mathcal{M}_{1}(\Lambda)$ of all absolutely continuous measures w.r.t. the Lebesgue measure with density bounded by 1 :

$$
\mathcal{M}_{1}^{0}(\Lambda)=\left\{\pi \in \mathcal{M}_{1}(\Lambda): \pi(d u)=\rho(u) d u \quad \text { and } \quad 0 \leq \rho(u) \leq 1 \quad \text { a.e. }\right\}
$$


$\mathcal{M}_{1}^{0}(\Lambda)$ is a closed subset of $\mathcal{M}_{1}(\Lambda)$ endowed with the weak topology and $D\left([0, T], \mathcal{M}_{1}^{0}(\Lambda)\right)$ is a closed subset of $D\left([0, T], \mathcal{M}_{1}(\Lambda)\right)$ for the Skorohod topology. To state next theorem we need the following definition.

Definition Given a Lebesgue absolutely continuous measure $\rho(u) d u \in \mathcal{M}_{1}^{0}(\Lambda)$, a sequence of probability measures $\left(\mu^{N}\right)_{N \geq 0}$ on $\mathcal{S}_{N}$ is said to correspond to the macroscopic profile $\rho$ if, for any smooth function $G$ and $\delta>0$

$$
\lim _{N \rightarrow \infty} \mu^{N}\left\{\left|\frac{1}{N^{d}} \sum_{x \in \Lambda_{N}} G(x / N) \eta(x)-\int_{\Lambda} G(u) \rho(u) d u\right|>\delta\right\}=0
$$

Theorem 2.1 Let $d \geq 3$ and assume that $D(\rho)$ can be continuously extended to the closed interval $[0,1]$. Let $\mu^{N}$ be a sequence of probability measures on $\mathcal{S}_{N}$ corresponding to the initial profile $\rho_{0}$. Then, IP a.s. the sequence of probability measures $\left(Q_{\mu^{N}}\right)_{N \geq 0}$ is tight and all its limit points $Q^{*}$ are concentrated on $\rho(t, u) \mathrm{d} u$, whose densities are weak solutions of the equation (2.15). Moreover if $D(\cdot)$ is Lipschitz continuous for $\rho \in(0,1)$, then $\left(Q_{\mu^{N}}\right)_{N \geq 0}$ converges weakly, as $N \uparrow \infty$, to $Q^{*}$. This limit point is concentrated on the unique weak solution of equation (2.15).

Denote by $\nu_{s}^{\alpha, N}$ the unique invariant measure of the Markov process $\left(\eta_{t}\right)_{t \in[0, T]}$ with generator $N^{2} \mathcal{L}_{N}$. We have the following:

Theorem 2.2 Let $d \geq 3$, assume that $D(\rho)$ can be continuously extended to the closed interval $[0,1]$ and Lipschitz continuous for $\rho \in(0,1)$. For every continuous function $G: \Lambda \rightarrow \mathbb{R}$ and every $\delta>0$,

$$
\lim _{N \rightarrow \infty} \nu_{s}^{\alpha, N}\left\{\left|\frac{1}{N^{d}} \sum_{x \in \Lambda_{N}} G(x / N) \eta(x)-\int_{\Lambda} G(u) \bar{\rho}(u) d u\right|>\delta\right\}=0, \quad I P=1
$$

with $\bar{\rho}(\cdot)$ satisfying (2.24).

\section{Strategy of proof and basic estimates}

\subsection{The steps to prove Theorem 2.1}

To prove the hydrodynamic behavior of the system we follow the entropy method introduced by [GPV]. As explained in Section 1, since the reference invariant state is not explicitly known, we compute the entropy of the state of the process with respect to a product measure with slowly varying profile $\gamma(\cdot)$. We prove in Lemma 3.8 that, provided $\gamma(\cdot)$ is smooth enough, $C^{1}$ suffices, and takes the prescribed value $b(\cdot)$ at the boundary, the rate to which the entropy increases is of the order of the volume, $N^{d}$, i.e the same order of the entropy and for finite time $T$ this implies only a modification of the constant multiplying $N^{d}$.

We divide the proof of the hydrodynamic behavior in three steps: tightness of the measures $\left(Q_{\mu^{N}}\right)_{N \geq 1}$, energy estimates and identification of the support of $Q^{*}$ as weak solution of (2.15) with fixed boundary conditions. We then refer to $[\mathrm{KL}]$, Chapter IV, that presents arguments, by now standard, to deduce the hydrodynamic behavior of the empirical measures from the preceding results and the uniqueness of the weak solution of (2.15). We state without proving the first two steps, tightness of the measures and energy estimates. The proof of them can be easily derived from results already in the literature, which we refer to. 
Proposition 3.1 (Tightness) For almost any disorder configuration $\alpha \in \Sigma_{D}$, the sequence $\left(Q_{\mu^{N}}\right)_{N \geq 1}$ is tight and all its limit points $Q^{*}$ are concentrated on absolutely continuous paths $\pi(t, d u)=\rho(t, u) d u$ whose density $\rho$ is positive and bounded above by 1 :

$$
Q^{*}\{\pi: \pi(t, d u)=\rho(t, u) d u\}=1, \quad Q^{*}\{\pi: 0 \leq \rho(t, u) \leq 1\}=1
$$

Tightness for non gradient systems in contact with reservoirs is proven in a way similar to the one for non gradient systems with periodic boundary conditions, see $[\mathrm{KL}]$, Chapter 7 , Section 6 . The main difference relies on the fact that for systems in contact with reservoirs the invariant states are not product probability measures and some additional argument is required. This can be proven as in [LMS], Section 6.

In the next step we prove that for almost any disorder configuration $\alpha \in \Sigma_{D}$, every limit point $Q^{*}$ of the sequence $\left(Q_{\mu_{N}}\right)_{N \geq 1}$ is concentrated on paths whose densities $\rho$ satisfy $(2.22)$.

Proposition 3.2 For almost any disorder configuration $\alpha \in \Sigma_{D}$, every limit points $Q^{*}$ of the sequence $\left(Q_{\mu^{N}}\right)_{N \geq 1}$ is concentrated on the trajectories that satisfies (IB1).

The proof can be done applying arguments as in Proposition A.1.1. of [KLO]. However the latter proof requires an application of Feynman-Kac formula, for which we have to replace our dynamic (2.7) (cf. [FM]).

We then show that $I P$ - a.s. any limit point $Q^{*}$ is supported on densities $\rho$ satisfying (2.15) in the weak sense. For $\ell \in I N, x \in \Lambda_{N}$, with $-N+\ell \leq x_{1} \leq N-\ell$ denote by $\eta^{\ell}(x)$ the average density of $\eta$ in a cube of width $2 \ell+1$ centered at $x$

$$
\eta^{\ell}(x)=\frac{1}{(2 \ell+1)^{d}} \sum_{y:|y-x| \leq \ell} \eta(y) .
$$

For a function $G$ on $\Lambda, e \in \mathcal{E}, \partial_{e}^{N} G$ denotes the discrete (space) derivative in the direction $e$

$$
\left(\partial_{e}^{N} G\right)(x / N)=N[G((x+e) / N)-G(x / N)] \quad \text { with } \quad x \quad \text { and } \quad x+e \in \Lambda_{N}
$$

and to short notation we denote by $\partial_{k}^{N} G:=\partial_{e_{k}}^{N} G$ for $1 \leq k \leq d$.

Proposition 3.3 Assume that $D(\rho)$ defined in (2.17) can be continuously extended in $[0,1]$. Then, for almost any disorder configuration $\alpha \in \Sigma_{D}$, any function $G$ in $\mathcal{C}_{c}^{1,2}([0, T] \times \stackrel{\circ}{)}$ and any $\delta>0$, we have

$$
\limsup _{c \rightarrow 0} \limsup _{a \rightarrow 0} \limsup _{N \rightarrow \infty} \mathbf{P}_{\mu^{N}}\left(\left|\mathcal{B}_{a, c}^{G, N}\right| \geq \delta\right)=0
$$

where

$$
\begin{aligned}
& \mathcal{B}_{a, c}^{G, N}= N^{-d} \sum_{x \in \Lambda_{N}} G(T, x / N) \eta_{T}(x)-N^{-d} \sum_{x \in \Lambda_{N}} G(0, x / N) \eta_{0}(x)-N^{-d} \sum_{x \in \Lambda_{N}} \int_{0}^{T} \partial_{s} G(s, x / N) \eta_{s}(x) d s \\
&+\sum_{1 \leq k, m \leq d} \int_{0}^{T} d s N^{1-d} \sum_{x \in \Lambda_{N}}\left(\partial_{k}^{N} G\right)(s, x / N)\left\{D_{k, m}\left(\eta_{s}^{[a N]}(x)\right)\right. \\
&\left.\times\left\{(2 c)^{-1}\left[\eta_{s}^{[a N]}\left(x+c N e_{m}\right)-\eta_{s}^{[a N]}\left(x-c N e_{m}\right)\right]\right\}\right\} .
\end{aligned}
$$


The proof is given in Subsection 3.3.

The last step states that $I P$ - a.s., any limit points $Q^{*}$ of the sequence $\left(Q_{\mu^{N}}\right)_{N \geq 1}$ is concentrated on the trajectories with fixed density at the boundary and equal to $b(\cdot)$ :

Proposition 3.4 IP- a.s., any limit point $Q^{*}$ of the sequence $\left(Q_{\mu^{N}}\right)_{N \geq 1}$ is concentrated on the trajectories that satisfy (IB3).

The proof is given in Subsection 3.4.

\subsection{Basic estimates}

Lemma 3.5 (Ergodic lemma) Let $V: \Sigma_{D} \times \Lambda \rightarrow \mathbb{R}$ a bounded function, local with respect to the first variable and continuous with respect to the second variable, that is for any $\alpha \in \Sigma_{D}$ the function $u \rightarrow V(\alpha, u)$ is continuous and there exists an integer $\ell \geq 1$ such that for all $u \in \Lambda$ the support of $V(\cdot, u) \subset\{-\ell, \cdots, \ell\}^{d}$. Then

$$
\lim _{N \rightarrow \infty} N^{-d} \sum_{x \in \Lambda_{N}} \tau_{x} V(\alpha, x / N)=\int_{\Lambda} \mathbb{E}[V(\cdot, u)] d u \quad \mathbb{P} \text { a.s.. }
$$

Proof. We decompose the left hand side of the limit (3.6) in two parts

$$
\begin{aligned}
N^{-d} \sum_{x \in \Lambda_{N}} \tau_{x} V(\alpha, x / N) & =N^{-d} \sum_{x \in \Lambda_{N}}\left(\tau_{x} V(\alpha, x / N)-\mathbb{E}[V(\cdot, x / N)]\right) \\
& +N^{-d} \sum_{x \in \Lambda_{N}} \mathbb{E}[V(\cdot, x / N)]-\int_{\Lambda} \mathbb{E}[V(\cdot, u)] d u .
\end{aligned}
$$

By the stationary of $\mathbb{P}$ and the continuity of $u \rightarrow \mathbb{E}[V(\cdot, u)]$, the second term of the the right hand side of the last equality converges to 0 as $N \rightarrow \infty$. The first term converges to 0 , from Chebychef inequality and the classical method of moments usually used in the proof of strong law of large numbers.

We start recalling the definition of relative entropy, which is the main tool in the [GPV] approach. Let

$\nu_{\rho(\cdot)}^{\alpha, N}$ be the product measure defined in (2.4) and $\mu$ a probability measure on $\mathcal{S}_{N}$. Denote by $H\left(\mu \mid \nu_{\rho(\cdot)}^{\alpha, N}\right)$ the relative entropy of $\mu$ with respect to $\nu_{\rho(\cdot)}^{\alpha, N}$ :

$$
H\left(\mu \mid \nu_{\rho(\cdot)}^{\alpha, N}\right)=\sup _{f}\left\{\int f(\eta) \mu(d \eta)-\log \int e^{f(\eta)} \nu_{\rho(\cdot)}^{\alpha, N}(d \eta)\right\}
$$

where the supremum is carried over all bounded functions on $\mathcal{S}_{N}$. Since $\nu_{\rho(\cdot)}^{\alpha, N}$ gives a positive probability to each configuration, $\mu$ is absolutely continuous with respect to $\nu_{\rho(\cdot)}^{\alpha, N}$ and we have an explicit formula for the entropy:

$$
H\left(\mu \mid \nu_{\rho(\cdot)}^{\alpha, N}\right)=\int \log \left\{\frac{d \mu}{d \nu_{\rho(\cdot)}^{\alpha, N}}\right\} d \mu
$$

Further, since there is at most one particle per site, there exists a constant $C$, that depends only on $\rho(\cdot)$, such that for all $\alpha$

$$
H\left(\mu \mid \nu_{\rho(\cdot)}^{\alpha, N}\right) \leq C N^{d}
$$


for all probability measures $\mu$ on $\mathcal{S}_{N}$ (cf. comments following Remark V.5.6 in [KL]).

It is well known that one of the main step in the derivation of hydrodynamic limit for the empirical density is a super exponential estimate which allows the replacement of local functions by functionals of the empirical density. One needs to estimate expression such as $\langle Z, f\rangle_{\mu^{N}}$ in terms of Dirichlet form $<-\mathcal{L}_{N} \sqrt{f(\eta)}, \sqrt{f(\eta)}>_{\mu^{N}}$, where $Z$ is a local function and $\left\langle\cdot, \cdot>_{\mu^{N}}\right.$ represents a scalar product with respect to some state $\mu^{N}$. Since in the context of boundary driven process the invariant state is not explicitly known and we fix as reference measure some product measure $\nu$, see Lemma 3.6, there are no reasons for $<-\mathcal{L}_{N} \sqrt{f(\eta)}, \sqrt{f(\eta)}>_{\nu}$ to be positive. Next lemma shows that this expression is almost positive. Let $\mathcal{D}_{N}^{0}(\cdot, \nu), \mathcal{D}_{N}^{b}(\cdot, \nu)$ be functionals from $h \in L^{2}(\nu)$ to $\mathbb{R}^{+}$:

$$
\begin{aligned}
& \mathcal{D}_{N}^{0}(h, \nu)=\frac{1}{2} \sum_{e \in \mathcal{E}} \sum_{x, x+e \in \Lambda_{N}} \int C_{N}(x, x+e ; \eta)\left(h\left(\eta^{x, x+e}\right)-h(\eta)\right)^{2} d \nu(\eta), \\
& \mathcal{D}_{N}^{b}(h, \nu)=\frac{1}{2} \sum_{x \in \Gamma_{N}} \int C^{b}(x / N, \eta)\left(h\left(\eta^{x}\right)-h(\eta)\right)^{2} d \nu(\eta) .
\end{aligned}
$$

Lemma 3.6 Let $\gamma: \Lambda \rightarrow(0,1)$ be a smooth function such that $\left.\gamma\right|_{\Gamma}=b(\cdot)$. For any $\alpha \in \Sigma_{D}$ and $a>0$ there exists a positive constant $C_{0} \equiv C_{0}\left(A,\|\nabla \gamma\|_{\infty}\right)$ so that for any $f \in L^{2}\left(\nu_{\gamma(\cdot)}^{\alpha, N}\right)$,

$$
\begin{gathered}
\int_{\mathcal{S}_{N}} f(\eta) \mathcal{L}_{N}^{0} f(\eta) d \nu_{\gamma(\cdot)}^{\alpha, N}(\eta) \leq-\left(1-\frac{1}{2 a}\right) \mathcal{D}_{N}^{0}\left(f, \nu_{\gamma(\cdot)}^{\alpha, N}\right)+C_{0} N^{d-2}(a+1)\|f\|_{L^{2}\left(\nu_{\gamma(\cdot)}^{\alpha, N}\right)}^{2} \\
\int_{\mathcal{S}_{N}} f(\eta) \mathcal{L}_{N}^{b} f(\eta) d \nu_{\gamma(\cdot)}^{\alpha, N}(\eta)=-\mathcal{D}_{N}^{b}\left(f, \nu_{\gamma(\cdot)}^{\alpha, N}\right)
\end{gathered}
$$

Proof. By (3.9),

$$
\begin{aligned}
\int_{\mathcal{S}_{N}} f(\eta) & \mathcal{L}_{N}^{0} f(\eta) d \nu_{\gamma(\cdot)}^{\alpha, N}(\eta)=-\mathcal{D}_{N}^{0}\left(f, \nu_{\gamma(\cdot)}^{\alpha, N}\right) \\
& +\frac{1}{2} \sum_{e \in \mathcal{E}} \sum_{x, x+e \in \Lambda_{N}} \int C_{N}(x, x+e ; \eta)\left(\nabla_{x, x+e} f\right)(\eta) f\left(\eta^{x, x+e}\right) R_{1}(x, x+e ; \eta) d \nu_{\gamma(\cdot)}^{\alpha, N}(\eta),
\end{aligned}
$$

where

$$
R_{1}(x, x+e ; \eta)=\left(\nabla_{x, x+e} \eta(x)\right)\left(e^{\left(N^{-1} \partial_{e}^{N} \lambda_{0}(\gamma(x / N))\right)}-1\right) .
$$

By the elementary inequality $2 u v \leq a u^{2}+a^{-1} v^{2}$ which holds for any $a>0$, for any $x, x+e \in \Lambda_{N}$

$$
\begin{aligned}
& \int C_{N}(x, x+e ; \eta)\left(\nabla_{x, x+e} f\right) f\left(\eta^{x, x+e}\right) R_{1}(x, x+e, \eta) d \nu_{\gamma(\cdot)}^{\alpha, N}(\eta) \\
& \quad \leq \frac{1}{2 a} \int C_{N}(x, x+e ; \eta)\left(\nabla_{x, x+e} f\right)^{2} d \nu_{\gamma(\cdot)}^{\alpha, N}(\eta)+\frac{a}{2} \int C_{N}(x, x+e ; \eta) f\left(\eta^{x, x+e}\right)^{2}\left(R_{1}(x, x+e)\right)^{2} d \nu_{\gamma(\cdot)}^{\alpha, N}(\eta) .
\end{aligned}
$$

To conclude the proof it remains to use Taylor expansion and an integration by part in the second term of the right hand side of the last inequality. On the other hand, since $\left.\gamma\right|_{\Gamma}=b(\cdot)$ the measure $\nu_{\gamma(\cdot)}^{\alpha, N}$ is reversible with respect to $\mathcal{L}_{N}^{b}$. A simple computation shows that

$$
\int_{\mathcal{S}_{N}} f(\eta) \mathcal{L}_{N}^{b} f(\eta) d \nu_{\gamma(\cdot)}^{\alpha, N}(\eta)=-\mathcal{D}_{N}^{b}\left(f, \nu_{\gamma(\cdot)}^{\alpha, N}\right)
$$


Lemma 3.7 Let $\rho, \rho_{0}: \Lambda \rightarrow(0,1)$ be two smooth functions. There exists a positive constant $C_{0}^{\prime} \equiv$ $C_{0}^{\prime}\left(A,\left\|\nabla \rho_{0}\right\|_{\infty},\|\nabla \rho\|_{\infty}\right)$ such that for any probability measure $\mu^{N}$ on $\mathcal{S}_{N}$ and for any $\alpha \in \Sigma_{D}$,

$$
\mathcal{D}_{N}^{0}\left(\sqrt{\frac{d \mu^{N}}{d \nu_{\rho(\cdot)}^{\alpha, N}}}, \nu_{\rho(\cdot)}^{\alpha, N}\right) \leq 2 \mathcal{D}_{N}^{0}\left(\sqrt{\frac{d \mu^{N}}{d \nu_{\rho_{0}(\cdot)}^{\alpha, N}}}, \nu_{\rho_{0}(\cdot)}^{\alpha, N}\right)+C_{0}^{\prime} N^{d-2} .
$$

Proof. Denote by $f(\eta)=\frac{d \mu^{N}}{d \nu_{\rho(\cdot)}^{\alpha, N}}(\eta)$ and $h(\eta)=\frac{d \mu^{N}}{d \nu_{\rho_{0}(\cdot)}^{\alpha, N}}(\eta)$. Since $f(\eta)=h(\eta) \frac{d \nu_{\rho_{0} \cdot(\cdot)}^{\alpha, N}(\eta)}{d \nu_{\rho(\cdot)}^{\alpha, N}(\eta)}$ we obtain for $e \in \mathcal{E}$ and $x, x+e \in \Lambda_{N}$ the following

$$
\begin{aligned}
& \int_{\mathcal{S}_{N}} C_{N}(x, x+e ; \eta)\left[\nabla_{x, x+e} \sqrt{f}(\eta)\right]^{2} d \nu_{\rho(\cdot)}^{\alpha, N}(\eta) \\
& \quad=\int_{\mathcal{S}_{N}} C_{N}(x, x+e ; \eta)\left[\sqrt{h}\left(\eta^{x, x+e}\right) R_{2}(x, x+e ; \eta)+\nabla_{x, x+e} \sqrt{h}(\eta)\right]^{2} d \nu_{\rho_{0}(\cdot)}^{\alpha, N}(\eta) \\
& \quad \leq 2 \int_{\mathcal{S}_{N}} C_{N}(x, x+e ; \eta)\left[\nabla_{x, x+e} \sqrt{h}(\eta)\right]^{2} d \nu_{\rho_{0}(\cdot)}^{\alpha, N}(\eta) \\
& \quad+2 \int_{\mathcal{S}_{N}} C_{N}(x, x+e ; \eta) h\left(\eta^{x, x+e}\right)\left[R_{2}(x, x+e ; \eta)\right]^{2} d \nu_{\rho_{0}(\cdot)}^{\alpha, N}(\eta)
\end{aligned}
$$

where

$$
R_{2}(x, x+e ; \eta)=\exp \left\{(1 / 2) N^{-1} \partial_{e}^{N}\left[\lambda_{0}(\rho(x / N))-\lambda_{0}\left(\rho_{0}(x / N)\right)\right] \nabla_{x, x+e} \eta(x)\right\}-1 .
$$

We conclude the proof using Taylor expansion and integration by parts.

Denote by $S_{t}^{N}$ the semigroup associated to the generator $N^{2} \mathcal{L}_{N}$. Given a probability measures $\mu^{N}$ on $\mathcal{S}_{N}$ denote by $\mu^{N}(t)$ the state of the process at time $t: \mu^{N}(t)=\mu^{N} S_{t}^{N}$.

Recall that $\gamma: \Lambda \rightarrow(0,1)$ is a smooth profile equal to $b$ at the boundary of $\Lambda$. Let $h_{t}^{N}$ be the density of $\mu^{N}(t)$ with respect to $\nu_{\gamma(\cdot)}^{\alpha, N}$. Let $\mathcal{L}_{\gamma, N}^{*}$ be the adjoint of $\mathcal{L}_{N}$ in $L^{2}\left(\nu_{\gamma(\cdot)}^{\alpha, N}\right)$. It is easy to check that

$$
\partial_{t} h_{t}^{N}=N^{2} \mathcal{L}_{\gamma, N}^{*} h_{t}^{N}
$$

Notice that $\mathcal{L}_{\gamma, N}^{*}$ is not a generator because $\nu_{\gamma(\cdot)}^{\alpha, N}$ is not an invariant measure for the Markov process with generator $\mathcal{L}_{N}$. We denote by $H_{N}(t)$ the entropy of $\mu^{N}(t)$ with respect to $\nu_{\gamma(\cdot)}^{\alpha, N}$, see (3.7),

$$
H_{N}(t):=H\left(\mu^{N}(t) \mid \nu_{\gamma(\cdot)}^{\alpha, N}\right)
$$

Lemma 3.8 There exists positive constant $C=C\left(\|\nabla \gamma\|_{\infty}\right)$ such that for any $a>0$ and for any $\alpha \in \Sigma_{D}$

$$
\partial_{t} H_{N}(t) \leq-2(1-a) N^{2} \mathcal{D}_{N}^{0}\left(\sqrt{h_{t}^{N}}, \nu_{\gamma(\cdot)}^{\alpha, N}\right)-2 N^{2} \mathcal{D}_{N}^{b}\left(\sqrt{h_{t}^{N}}, \nu_{\gamma(\cdot)}^{\alpha, N}\right)+\frac{C}{a} N^{d}
$$

Proof. By (3.13) and the explicit formula for the entropy we have that

$$
\partial_{t} H_{N}(t)=N^{2} \int_{\mathcal{S}_{N}} h_{t}^{N} \mathcal{L}_{N} \log \left(h_{t}^{N}\right) d \nu_{\gamma(\cdot)}^{\alpha, N}
$$


Using the basic inequality $a(\log b-\log a) \leq-(\sqrt{a}-\sqrt{b})^{2}+(b-a)$ for positive $a$ and $b$, we obtain

$$
\begin{aligned}
\partial_{t} H_{N}(t) \leq & -2 N^{2} \mathcal{D}_{N}^{0}\left(\sqrt{h_{t}^{N}}, \nu_{\gamma(\cdot)}^{\alpha, N}\right)-2 N^{2} \mathcal{D}_{N}^{b}\left(\sqrt{h_{t}^{N}}, \nu_{\gamma(\cdot)}^{\alpha, N}\right) \\
& +N^{2} \int_{\mathcal{S}_{N}} \mathcal{L}_{N}^{0} h_{t}^{N} d \nu_{\gamma(\cdot)}^{\alpha, N}+N^{2} \int_{\mathcal{S}_{N}} \mathcal{L}_{N}^{b} h_{t}^{N} d \nu_{\gamma(\cdot)}^{\alpha, N}
\end{aligned}
$$

Since $\gamma(u)=b(u)$ for $u \in \Gamma, \nu_{\gamma(\cdot)}^{\alpha, N}$ is reversible with respect to $\mathcal{L}_{N}^{b}$. This implies that

$$
\int_{\mathcal{S}_{N}} \mathcal{L}_{N}^{b} h_{t}^{N} d \nu_{\gamma(\cdot)}^{\alpha, N}=0
$$

We shall now obtain a bound for $\int_{\mathcal{S}_{N}} \mathcal{L}_{N}^{0} h_{t}^{N} d \nu_{\gamma(\cdot)}^{\alpha, N}$ in terms of $\mathcal{D}_{N}^{0}$. Denote by $R: \mathbb{R} \rightarrow \mathbb{R}$ the function defined by $R(u)=e^{u}-1-u$. A standard computation shows that

$$
\begin{aligned}
& N^{2} \int_{\mathcal{S}_{N}} \mathcal{L}_{N}^{0} h_{t}^{N} d \nu_{\gamma(\cdot)}^{\alpha, N} \\
& =N^{2} \sum_{e \in \mathcal{E}} \sum_{x, x+e \in \Lambda_{N}} \int C_{N}(x, x+e ; \eta) h_{t}^{N}(\eta) R\left(N^{-1} \partial_{e}^{N} \lambda_{0}(\gamma(x / N)) \nabla_{x, x+e} \eta(x)\right) d \nu_{\gamma(\cdot)}^{\alpha, N}(\eta) \\
& \quad+N \sum_{e \in \mathcal{E}} \sum_{x, x+e \in \Lambda_{N}}\left(\partial_{e}^{N} \lambda_{0}(\gamma(x / N)) \int W_{x, x+e}(\eta) h_{t}^{N}(\eta) d \nu_{\gamma(\cdot)}^{\alpha, N}(\eta)\right.
\end{aligned}
$$

where $W_{x, x+e}(\eta)$ is the current over the bond $(x, x+e)$ :

$$
W_{x, x+e}(\eta) \equiv C_{N}(x, x+e ; \eta)[\eta(x)-\eta(x+e)]
$$

We will often omit to write the dependence of $W_{x, x+e}(\eta)$ on $N$ and $\eta$. By Taylor expansion and the elementary inequality $|R(u)| \leq \frac{u^{2}}{2} e^{|u|}$, we obtain using the fact that $\gamma$ is smooth and $h_{t}^{N}$ is a probability density with respect to $\nu_{\gamma(\cdot)}^{\alpha, N}$, that the first term of the right hand side of the (3.16) is bounded by $C N^{d}$ for some positive constant $C$. On the other hand integrating by part, applying the same computations as in Lemma 5.1 of [LMS], we obtain that there exists a constant $C_{0}=C\left(\|\nabla \gamma\|_{\infty}\right)$ so that for any $a>0$

$$
\int W_{x, x+e} h_{t}^{N} d \nu_{\gamma(\cdot)}^{\alpha, N} \leq \frac{1}{a} \int C_{N}(x, x+e ; \eta)\left(\nabla_{x, x+e} \sqrt{h_{t}^{N}}\right)^{2} d \nu_{\gamma(\cdot)}^{\alpha, N}+C_{0}\left\{a+N^{-1}\right\}
$$

for $x, x+e \in \Lambda_{N}$.

For $z \in \Lambda_{N}, M \in I N$ denote by $\Lambda_{M}(z)$ the intersection of a cube centered at $z \in \Lambda_{N}$ of edge $2 M+1$ with $\Lambda_{N}$, i.e

$$
\Lambda_{M}(z):=\left\{z+\Lambda_{M}\right\} \cap \Lambda_{N}
$$

For probability measure $\nu^{N}$ on $\mathcal{S}_{N}$, denote by $\mathcal{D}_{M, z}^{0}\left(\cdot, \nu^{N}\right)$ the Dirichlet form corresponding to jumps in $\Lambda_{M}(z)$ :

$$
\mathcal{D}_{M, z}^{0}\left(f, \nu^{N}\right)=\frac{1}{2} \sum_{x, x+e \in \Lambda_{M}(z)} \int C_{N}(x, x+e ; \eta)\left(\nabla_{x, x+e} f(\eta)\right)^{2} d \nu^{N}(\eta)
$$


Similarly, for $z \in \Gamma_{N}$ define $\mathcal{D}_{M, z}^{b}\left(\cdot, \nu^{N}\right)$ the Dirichlet form corresponding to creation and destruction of particles at sites in $\Gamma_{N}$ which are at distance less than $M$ from $z$ :

$$
\mathcal{D}_{M, z}^{b}\left(f, \nu^{N}\right)=\frac{1}{2} \sum_{x \in \Gamma_{N} \cap \Lambda_{M}(z)} \int C^{b}(x / N, \eta)\left(f\left(\eta^{x}\right)-f(\eta)\right)^{2} d \nu^{N}(\eta) .
$$

Fix any $z \in \Gamma_{N}$ denote by $f_{t}^{z, N}$ the Radon-Nikodym derivative of $\mu^{N}(t)$ with respect to $\nu_{b(z / N)}^{\alpha, N}$, the random Bernoulli measure on $\mathcal{S}_{N}$ with constant parameter equal to $b\left(\frac{z}{N}\right)$. Recall that we denoted by $h_{t}^{N}$ the RadonNikodym derivative of $\mu^{N}(t)$ with respect to $\nu_{\gamma(\cdot)}^{\alpha, N}$ and that $b\left(\frac{z}{N}\right)=\gamma\left(\frac{z}{N}\right)$ for $z \in \Gamma$. We have the following result.

Lemma 3.9 Take $M \in I N, M<N$. There exists a positive constant $C_{0}=C\left(\|\nabla \gamma\|_{\infty}\right)$ depending only on $\gamma(\cdot)$ such that for any $z \in \Gamma_{N}$

$$
\begin{aligned}
& \mathcal{D}_{M, z}^{0}\left(\sqrt{f_{t}^{z, N}}, \nu_{b(z / N)}^{\alpha, N}\right) \leq 2 \mathcal{D}_{M, z}^{0}\left(\sqrt{h_{t}^{N}}, \nu_{\gamma(\cdot)}^{\alpha, N}\right)+C_{0} \frac{M^{d}}{N^{2}} \\
& \mathcal{D}_{M, z}^{b}\left(\sqrt{f_{t}^{z, N}}, \nu_{b(z / N)}^{\alpha, N}\right) \leq 2 \mathcal{D}_{M, z}^{b}\left(\sqrt{h_{t}^{N}}, \nu_{\gamma(\cdot)}^{\alpha, N}\right)+C_{0} \frac{M^{d+1}}{N^{2}}
\end{aligned}
$$

The proof is similar to the proof of Lemma 3.7.

\subsection{Proof of Proposition 3.3}

We prove in this section Proposition 3.3. Let $Q^{*}$ be a limit point of the sequence $\left(Q_{\mu^{N}}\right)_{N \geq 1}$ and assume, without loss of generality, that $\mathbb{P}$ - a.s., $Q_{\mu^{N}}$ converges to $Q^{*}$. Fix a function $G$ in $\mathcal{C}_{c}^{1,2}\left([0, T] \times \Lambda^{\prime}\right.$. For $\alpha \in \Omega_{D}$ consider the $\mathbf{P}_{\mu^{N}}$ martingales with respect to the natural filtration associated with $\left(\eta_{t}\right)_{t \in[0, T]}$, $M_{t}^{G} \equiv M_{t}^{G, N, \alpha}$ and $\mathcal{N}_{t}^{G} \equiv \mathcal{N}_{t}^{G, N, \alpha}, t \in[0, T]$, defined by

$$
\begin{aligned}
& M_{t}^{G}=<\pi_{t}^{N}, G_{t}>-<\pi_{0}^{N}, G_{0}>-\int_{0}^{t}\left(<\pi_{s}^{N}, \partial_{s} G_{s}>+N^{2} \mathcal{L}_{N}<\pi_{s}^{N}, G_{s}>\right) d s \\
& \mathcal{N}_{t}^{G}=\left(M_{t}^{G}\right)^{2}-\int_{0}^{t}\left\{N^{2} \mathcal{L}_{N}^{\alpha}\left(<\pi_{s}^{N}, G_{s}>\right)^{2}-2<\pi_{s}^{N}, G_{s}>N^{2} \mathcal{L}_{N}<\pi_{s}^{N}, G_{s}>\right\} d s .
\end{aligned}
$$

A computation of the integral term of $\mathcal{N}_{t}^{G}$ shows that the expectation of the quadratic variation of $M_{t}^{G}$ vanishes as $N \uparrow 0$. Therefore, by Doob's inequality, for every $\delta>0, I P=1$,

$$
\lim _{N \rightarrow \infty} \mathbf{P}_{\mu_{N}}\left[\sup _{0 \leq t \leq T}\left|M_{t}^{G}\right|>\delta\right]=0
$$

Thanks to (2.13) and since for any $s \in[0, T]$ the function $G_{s}$ has compact support in $\Lambda$, a summation by parts permits to rewrite the integral term of $M_{t}^{G}$ as

$$
\int_{0}^{t}<\pi_{s}^{N}, \partial_{s} G_{s}>d s+\int_{0}^{t}\left\{N^{1-d} \sum_{k=1}^{d} \sum_{x \in \Lambda_{N}}\left(\partial_{k}^{N} G_{s}\right)(x / N) W_{x, x+e_{k}}\left(\eta_{s}\right)\right\} d s,
$$


where the current $W_{x, x+e_{k}}$ is defined in (3.17). To localize the dynamics define for any $0<r<1$

$$
\begin{aligned}
& \Lambda_{r}=[-r, r] \times \mathbb{T}^{d-1}, \quad \Lambda_{r N}=\left\{\left(x_{1}, \cdots, x_{d}\right) \in \Lambda_{N}: \quad-r N \leq x_{1} \leq r N\right\} \\
& \Gamma_{r N}=\left\{x \in \Lambda_{r N}: \quad x_{1}= \pm r N\right\} .
\end{aligned}
$$

Set, for $0<a<c<1, k=1, \ldots, d$,

$$
\mathbb{V}_{k}^{N, c, a}(\eta, \alpha)=N W_{0, e_{k}}+\sum_{m=1}^{d} D_{k, m}\left(\eta^{[a N]}(0)\right)\left\{(2 c)^{-1}\left[\eta^{[a N]}\left(c N e_{m}\right)-\eta^{[a N]}\left(-c N e_{m}\right)\right]\right\}
$$

Next theorem is the main step in the proof of Proposition 3.3.

Theorem 3.10 Assume that $D(\cdot)$ defined in (2.17) can be continuously extended in $[0,1]$. Then, $\mathbb{P}=1$, for any $G \in \mathcal{C}_{c}^{1,2}([0, T] \times \stackrel{\circ}{\Lambda}$,

$$
\underset{c \rightarrow 0}{\limsup \limsup } \limsup _{a \rightarrow 0} \mathbf{E}_{\mu_{N}}\left[\left|N^{-d} \int_{0}^{T} \sum_{x \in \Lambda_{N}} G_{s}(x / N) \tau_{x} \mathbf{V}_{k}^{N, c, a}\left(\eta_{s}, \alpha\right) d s\right|\right]=0
$$

for $k=1, \ldots, d$.

Proof. Let $0<\theta<1$ such that for any $t \in[0, T]$, the support of the function $G_{t}$ is a subset of $\Lambda_{(1-2 \theta)}$. Fix a smooth function $\gamma_{\theta}: \Lambda \rightarrow(0,1)$ which coincides with $b$ at the boundary of $\Lambda$ and constant inside $\Lambda_{(1-\theta)}$. Denote by $Z_{k}^{N, c, a}(G, \eta)$ the quantity

$$
Z_{k}^{N, c, a}(G, \eta)=N^{-d} \sum_{x \in \Lambda_{N}} G(x / N) \tau_{x} \mathbf{V}_{k}^{N, c, a}\left(\eta_{s}, \alpha\right)
$$

Since the entropy of $\mu^{N}$ with respect to $\nu_{\gamma_{\theta}(\cdot)}^{\alpha, N}$ is bounded by $C_{\theta}\left|\Lambda_{N}\right|$ for some finite constant $C_{\theta}$, by the entropy inequality, the left hand side of (3.26) is bounded above by

$$
\frac{C_{\theta}}{B}+\frac{1}{B N^{d}} \log \mathbf{E}_{\nu_{\gamma_{\theta}(\cdot)}^{\alpha, N}}\left[\exp \left\{B N^{d}\left|\int_{0}^{T} Z_{k}^{N, a, c}\left(G_{s}, \eta_{s}\right) d s\right|\right\}\right]
$$

for any positive $B$. Since $e^{|x|} \leq e^{x}+e^{-x}$ and $\lim \sup N^{-d} \log \left\{a_{N}+b_{N}\right\} \leq \max \left\{\lim \sup N^{-d} \log a_{N}\right.$, $\limsup N^{-d} \log b_{N}$ \}, we may remove the absolute value in the second term of (3.27), provided our estimate remains in force if we replace $G$ by $-G$. By the Feynman-Kac formula,

$$
\frac{1}{B N^{d}} \log \mathbf{E}_{\nu_{\gamma_{\theta}(\cdot)}^{\alpha, N}}\left[\exp \left\{B N^{d} \int_{0}^{T} Z_{k}^{N, a, c}\left(G_{s}, \eta_{s}\right) d s\right\}\right] \leq \frac{1}{B N^{d}} \int_{0}^{T} \lambda_{N, c, a}\left(G_{s}\right) d s
$$

where $\lambda_{N, c, a}\left(G_{s}\right)$ is the largest eigenvalue of the $N^{2}\left\{\mathcal{L}_{N}^{\text {sym }}+B Z_{k}^{N, c, a}\left(G_{s}, \eta\right)\right\}$ where $\mathcal{L}_{N}^{\text {sym }}:=\frac{1}{2}\left(\mathcal{L}_{N}+\mathcal{L}_{\gamma_{\theta}, N}^{*}\right)$ and $\mathcal{L}_{\gamma_{\theta}, N}^{*}$ is the adjoint of $\mathcal{L}_{N}$ in $L^{2}\left(\nu_{\gamma_{\theta}(\cdot)}^{\alpha, N}\right)$. By the variational formula for the largest eigenvalue, for $s \in[0, T]$, we have that

$$
\frac{1}{B N^{d}} \lambda_{N, c, a}\left(G_{s}\right)=\sup _{f}\left\{\int Z_{k}^{N, c, a}\left(G_{s}, \eta\right) f(\eta) \nu_{\gamma_{\theta}(\cdot)}^{\alpha, N}(d \eta)+\frac{N^{2-d}}{B}<\mathcal{L}_{N} \sqrt{f}, \sqrt{f}>_{\gamma_{\theta}(\cdot)}\right\} .
$$


In this formula the supremum is carried over all densities $f$ with respect to $\nu_{\gamma_{\theta}(\cdot)}^{\alpha, N}$ and notice that we used $<\mathcal{L}_{N} \sqrt{f}, \sqrt{f}>_{\gamma_{\theta}(\cdot)}=<\mathcal{L}_{N}^{s y m} \sqrt{f}, \sqrt{f}>_{\gamma_{\theta}(\cdot)}$. Since $\gamma_{\theta}(\cdot)$ coincides with $b(\cdot)$ on $\Gamma, \mathcal{L}_{N}^{b}$ is reversible with respect to $\gamma_{\theta}(\cdot)$, so that $\left\langle\mathcal{L}_{N}^{b} \sqrt{f}, \sqrt{f}>_{\gamma_{\theta}(\cdot)}\right.$ is negative. We then apply simply (3.10) of Lemma 3.6 with $a=1$ to estimate $\left\langle\mathcal{L}_{N} \sqrt{f}, \sqrt{f}>_{\gamma_{\theta}(\cdot)}\right.$ by $-(1 / 2) \mathcal{D}_{N}^{0}\left(\sqrt{f}, \nu_{\gamma_{\theta}(\cdot)}^{\alpha, N}\right)+C_{\theta}^{\prime} N^{d-2}$ for some constant $C_{\theta}^{\prime}$. In particular, to prove the theorem, we just need to show that

$$
\limsup _{c \rightarrow 0} \limsup _{a \rightarrow 0} \limsup _{N \rightarrow \infty} \int_{0}^{T} d s \sup _{f}\left\{\int Z_{k}^{N, c, a}\left(G_{s}, \eta\right) f(\eta) \nu_{\gamma_{\theta}(\cdot)}^{\alpha, N}(d \eta)-\frac{1}{B} N^{2-d} \mathcal{D}_{N}^{0}\left(\sqrt{f}, \nu_{\gamma_{\theta}}^{\alpha, N}\right)\right\}=0
$$

for every $B>0$ and then let $B \uparrow \infty$. Notice that for $N$ large enough and $a, c$ small enough, the function $Z_{k}^{N, c, a}\left(G_{s}, \eta\right)$ depends on the configuration $\eta$ only through the variables $\left\{\eta(x), x \in \Lambda_{(1-\theta) N}\right\}$. Since $\gamma_{\theta}(\cdot)$ is constant, say equal to $\gamma_{0}$ in $\Lambda_{(1-\theta)}$, we may replace $\nu_{\gamma_{\theta}(\cdot)}^{\alpha, N}$ in the previous formula by $\nu_{\gamma_{0}}^{\alpha, N}$. The $\nu_{\gamma_{0}}^{\alpha, N}$ is reversible for $\mathcal{L}_{N}^{0}$ and therefore $\mathcal{D}_{N}^{0}\left(\cdot, \nu_{\gamma_{0}}^{\alpha, N}\right)$ is the Dirichlet form associated to the generator $\mathcal{L}_{N}^{0}$. Since the Dirichlet form is convex, it remains to show that

$$
\limsup _{c \rightarrow 0} \limsup _{a \rightarrow 0} \limsup _{N \rightarrow \infty} \int_{0}^{T} d s \sup _{f}\left\{\int Z_{k}^{N, c, a}\left(G_{s}, \eta\right) f(\eta) \nu_{\gamma_{0}(\cdot)}^{\alpha, N}(d \eta)-\frac{1}{B} N^{2-d} \mathcal{D}_{N}^{0}\left(\sqrt{f}, \nu_{\gamma_{0}}^{\alpha, N}\right)\right\}=0
$$

for every $B>0$. This result has been proved in [FM], Theorem 3.2.

Proof of Proposition 3.3: By (3.21), (3.23) and (3.25), applying Theorem 3.10 we obtain (3.4).

\subsection{Proof of Proposition 3.4}

For $a>0, u \in \Lambda$ denote

$$
\iota_{a}(u)=\frac{1}{\left|[-a, a]^{d} \cap \Lambda\right|} \mathbb{I}_{\left\{[-a, a]^{d} \cap \Lambda\right\}}(u) ;
$$

and for $A \subset \Lambda$ define the sets $A^{ \pm}$as

$$
A^{+}=\left\{\left(u_{1}, \ldots, u_{d}\right) \in A \quad: \quad u_{1}>0\right\}, \quad A^{-}=\left\{\left(u_{1}, \ldots, u_{d}\right) \in A \quad: \quad u_{1}<0\right\} .
$$

We define similarly $A_{N}^{+}$and $A_{N}^{-}$when $A_{N} \subset \Lambda_{N}$. Let $G(\cdot, \cdot) \in C^{1,2}([0, T] \times \Lambda), \mu \in D\left([0, T], \mathcal{M}_{1}(\Lambda)\right)$ and for $0<a<c<1$, define the following functional

$$
\begin{aligned}
\hat{F}_{a, c}^{G}(\mu(\cdot, \cdot))= & \int_{0}^{T} d s \int_{\Lambda(1-c)} d u\left\{G_{s}(u)(2 c)^{-1}\left[\left(\mu_{s} \star \iota_{a}\right)\left(u+c e_{1}\right)-\left(\mu_{s} \star \iota_{a}\right)\left(u-c e_{1}\right)\right]\right\} \\
& +\int_{0}^{T} d s \int_{\Lambda} d u \partial_{e_{1}} G_{s}(u)\left(\mu_{s} \star \iota_{a}\right)(u)-\int_{0}^{T} d s\left\{\int_{\Gamma} b(u) \mathbf{n}_{1}(u) G_{s}(u) \mathrm{dS}\right\}
\end{aligned}
$$

where $G_{s}(u) \equiv G(s, u), \mathbf{n}=\left(\mathbf{n}_{1}, \ldots, \mathbf{n}_{d}\right)$ is the outward unit normal vector to the boundary surface $\Gamma$ and $\mathrm{d} S$ is the surface element of $\Gamma$. The proof of Proposition 3.4 follows from the next lemma.

Lemma 3.11 For $G(\cdot, \cdot) \in C^{1,2}([0, T] \times \Lambda), \mathbb{P}$ a.s. we have

$$
\limsup _{c \rightarrow 0} \limsup _{a \rightarrow 0} \limsup _{N \rightarrow \infty} \mathbf{E}^{Q_{\mu^{N}}}\left[\left|\hat{F}_{a, c}^{G}\left(\mu^{N}(\cdot, \cdot)\right)\right|\right]=0
$$


Proof. To short notation, denote $f_{s}(u):=\left(\mu_{s} \star \iota_{a}\right)(u)$. Taylor expanding we have that

$$
\begin{aligned}
& \int_{\Lambda(1-c)} d u\left\{G_{s}(u)(2 c)^{-1}\left[f_{s}\left(u+c e_{1}\right)-f_{s}\left(u-c e_{1}\right)\right]\right\} \\
& =\frac{1}{2 c} \int_{\left(\Lambda \backslash \Lambda_{(1-2 c)}\right)^{+}} G_{s}\left(u-c e_{1}\right) f_{s}(u) d u-\frac{1}{2 c} \int_{\left(\Lambda \backslash \Lambda_{(1-2 c)}\right)^{-}} G_{s}\left(u+c e_{1}\right) f_{s}(u) d u \\
& -\int_{\Lambda(1-2 c)} \partial_{e_{1}} G_{s}(u) f_{s}(u) d u+c \int_{\Lambda(1-2 c)} R(G, c, s, u) f_{s}(u) d u
\end{aligned}
$$

where $|R(G, c, s, u)| \leq \sup _{u \in \Lambda} \sup _{s \in[0, T]}\left|\partial_{e_{1}}^{2} G_{s}(\cdot)\right|$. Since $f_{s}(u) \leq 1$ uniformly in $s$ and $u$

$$
\left|\int_{\Lambda(1-c)} R(G, c, s, u) f_{s}(u) d u\right| \leq 2 \sup _{u \in \Lambda} \sup _{s \in[0, T]}\left|\partial_{e_{1}}^{2} G_{s}(u)\right|
$$

and

$$
\left|\int_{\Lambda(1-2 c)} \partial_{e_{1}} G_{s}(u) f_{s}(u) d u-\int_{\Lambda} \partial_{e_{1}} G_{s}(u) f_{s}(u) d u\right| \leq 2 c \sup _{u \in \Lambda} \sup _{s \in[0, T]}\left|\partial_{e_{1}} G_{s}(u)\right| .
$$

Taking in account (3.31), (3.28) and (3.32) the lemma is then proven once we show that $\mathbb{P}=1$ the following holds

$$
\begin{array}{r}
\limsup _{c \rightarrow 0} \limsup _{a \rightarrow 0} \limsup _{N \rightarrow \infty} \mathbf{E}_{\mu^{N}}\left[\mid \int_{0}^{T} d s\left\{\frac{1}{2 c N^{d}} \sum_{x \in\left(\Lambda_{(1-a) N} \backslash \Lambda_{(1-a-2 c) N}\right)^{ \pm}} G_{s}\left(\frac{x}{N}\right) \eta_{s}^{a N}(x)\right.\right. \\
\left.\left.-\frac{1}{N^{d-1}} \sum_{x \in \Gamma_{N}^{ \pm}} b\left(\frac{x}{N}\right) G_{s}\left(\frac{x}{N}\right)\right\} \mid\right]=0
\end{array}
$$

where for $0<\varepsilon<1, \Lambda_{\varepsilon N}$ and $\left(\Lambda_{\varepsilon N}\right)^{+}$are defined in (3.24) and below (3.29). By adding and subtracting the same quantity in the expectation of (3.33), it is easy to see that the limit (3.33) follows once the next two lemmas are proven.

Lemma 3.12 For $G(\cdot, \cdot) \in C^{1,2}([0, T] \times \Lambda), \mathbb{P}$ a.s. we have

$$
\begin{array}{r}
\lim _{\ell \rightarrow \infty} \limsup _{c \rightarrow 0} \limsup _{a \rightarrow 0} \limsup _{N \rightarrow \infty} \mathbf{E}_{\mu^{N}}\left[\mid \int_{0}^{T} d s\left\{\frac{1}{2 c N^{d}} \sum_{x \in\left(\Lambda_{(1-a) N} \backslash \Lambda_{(1-a-2 c) N}\right)^{ \pm}} G_{s}(x / N) \eta_{s}^{a N}(x)\right.\right. \\
\left.\left.-\frac{1}{N^{d-1}} \sum_{x \in \Gamma_{\left(1-\frac{\ell}{N}\right) N}^{ \pm}} G_{s}(x / N) \eta_{s}^{\ell}(x)\right\} \mid\right]=0 .
\end{array}
$$

Lemma 3.13 For $G(\cdot, \cdot) \in C^{1,2}([0, T] \times \Lambda), \mathbb{P}$ a.s. we have

$$
\begin{aligned}
\lim _{\ell \rightarrow \infty} \limsup _{N \rightarrow \infty} \mathbf{E}_{\mu^{N}}\left[\mid \int_{0}^{T} d s\left\{\frac{1}{N^{d-1}} \sum_{x \in \Gamma_{\left(1-\frac{\ell}{N}\right) N}^{ \pm}} G_{s}(x / N) \eta_{s}^{\ell}(x)\right.\right. \\
\\
\left.\left.\quad-\frac{1}{N^{d-1}} \sum_{x \in \Gamma_{N}^{ \pm}} b(x / N) G_{s}(x / N)\right\} \mid\right]=0 .
\end{aligned}
$$


Proof of Lemma 3.12. The summation in (3.34) contains two similar terms. We consider the one corresponding to the summation of the right hand side of $\Lambda_{N}$ (i.e. the one with signe + ). By Taylor expansion applied to the function $G$, the expectation in the statement of the lemma is bounded above by

$$
\mathbf{E}_{\mu^{N}}\left[\left|\int_{0}^{T} d s \frac{1}{N^{d-1}} \sum_{\check{x} \in \mathrm{T}_{N}^{d-1}} G_{s}\left(1, \frac{\check{x}}{N}\right)\left\{\frac{1}{2 c N} \sum_{x_{1}=N(1-a-2 c)+1}^{N(1-a)}\left(\eta_{s}^{a N}\left(x_{1}, \check{x}\right)-\eta_{s}^{\ell}(N-\ell, \check{x})\right)\right\}\right|\right]+R(N, a, c, G),
$$

where for $x_{1} \in[-N, N], \check{x}=\left(x_{2}, \cdots, x_{d}\right) \in \mathbb{T}_{N}^{d-1}$ the vector $\left(x_{1}, \check{x}\right)$ stands for the element $\left(x_{1}, x_{2}, \cdots, x_{d}\right) \in$ $\Lambda_{N}$. We denoted by $R(N, a, c, G)$ a quantity so that for $G \in C^{1,2}([0, T] \times \Lambda)$,

$$
\limsup _{c \rightarrow 0} \limsup _{a \rightarrow 0} \limsup _{N \rightarrow \infty}|R(N, a, c, G)|=0 .
$$

The next step consists in replacing the density average over a small macroscopic box of length $a N$ by a large microscopic box. More precisely, for $N$ large enough the expectation of the last quantity is bounded above by

$$
C\|G\|_{\infty} \sup _{2 \ell<|y| \leq 2 N c} \mathbf{E}_{\mu^{N}}\left[\int_{0}^{T} d s \frac{1}{N^{d-1}} \sum_{\check{x} \in \mathrm{T}_{N}^{d-1}}\left|\eta_{s}^{\ell}((N-\ell, \check{x})+y)-\eta_{s}^{(\ell)}(N-\ell, \check{x})\right|\right]+R(N, a, c, \ell),
$$

where for all $\ell, R(N, a, c, \ell)$ satisfy (3.36) and $C$ is a positive constant. Observe that the first term of the previous formula is not depending on $a$ but only on $c, N$ and $\ell$.

In view of the estimate (3.12) and Lemma 3.8 on the Dirichlet form $\mathcal{D}_{N}^{0}$ and the entropy, by the usual two blocks estimate, the first term of (3.37) converges to 0 an $N \uparrow \infty, c \downarrow 0$ and $\ell \uparrow \infty$. That concludes the proof of Lemma 3.12 .

Proof of Lemma 3.13. The summation in (3.35) contains two similar terms, we consider the one corresponding to the summation of the right hand side of $\Lambda_{N}$. It is easy to see that the expectation in (3.35) is bounded above by

$$
\|G\|_{\infty} \frac{1}{N^{d-1}} \sum_{y \in \Gamma_{N}^{+}} \mathbf{E}_{\mu^{N}}\left[\int_{0}^{T} d s\left|\eta_{s}^{\ell}\left(y-\ell e_{1}\right)-b(y / N)\right|\right] .
$$

For any fixed positive integer $\ell$ denote by $\Gamma_{0}^{\ell}=\left\{(0, \hat{x}): \quad \hat{x} \in \mathbb{T}_{N}^{d-1}, \quad|\hat{x}| \leq \ell\right\}=\left(\{0\} \times \mathbb{T}_{N}^{d-1}\right) \cap \Lambda_{\ell}(0)$, for notation see (3.18). For $u \in \Gamma$, denote

$$
\tilde{D}_{\ell, 0}^{b, u}(f, \nu)=\frac{1}{2} \sum_{x \in \Gamma_{0}^{\ell}} \int \tilde{C}_{0}^{b}(u, x, \eta)\left(f\left(\eta^{x}\right)-f(\eta)\right)^{2} d \nu(\eta)
$$

where

$$
\tilde{C}_{0}^{b}(u, x, \eta)=\eta(x) \exp \left\{-\frac{\alpha(x)+\lambda_{0}(b(u))}{2}\right\}+(1-\eta(x)) \exp \left\{\frac{\alpha(x)+\lambda_{0}(b(u))}{2}\right\} .
$$

The difference with the rate in (2.11) is that here $u$ is fixed. Let $\nu_{b(u)}^{\alpha, N}$ be the product measure, see (2.4), where $\rho\left(\frac{x}{N}\right) \equiv b(u)$ for $\forall x \in \Lambda_{N}$ and $\nu_{b(u)}^{\alpha, \ell}$ the restriction of $\nu_{b(u)}^{\alpha, N}$ to $\{0,1\}^{\Lambda_{\ell}(0)}$. Let $f: \mathcal{S}_{N} \rightarrow \mathbb{R}$, denote by $f^{\ell}$ the conditional expectation of $f$ with respect to the $\sigma$-algebra generated by $\left\{\eta(z): z \in \Lambda_{\ell}(0)\right\}$ :

$$
f^{\ell}(\xi)=\frac{1}{\nu_{b(u)}^{\alpha, \ell}(\xi)} \int \mathbb{I}_{\left\{\eta ; \eta(z)=\xi(z), z \in \Lambda_{\ell}(0)\right\}} f(\eta) d \nu_{b(u)}^{\alpha, N}(\eta) \quad \text { for all } \quad \xi \in\{0,1\}^{\Lambda_{\ell}(0)}
$$


Note that $\left|\eta^{\ell}(0)-b(u)\right|$ depends only on coordinates on the box $\Lambda_{\ell}(0)$, then by Fubini's Theorem,

$$
\mathbf{E}_{\mu^{N}}\left[\int_{0}^{T} d s\left|\eta_{s}^{\ell}\left(y-\ell e_{1}\right)-b(y / N)\right|\right]=T \int\left|\eta^{\ell}(0)-b(y / N)\right|\left(\tau_{-\left(y-\ell e_{1}\right)} \bar{f}_{T}^{y, N}\right)^{\ell}(\eta) d \nu_{b\left(\frac{y}{N}\right)}^{\alpha, \ell}(\eta)
$$

where $\bar{f}_{T}^{y, N}=\frac{1}{T} \int_{0}^{T} f_{s}^{y, N} d s$ and for all $0 \leq s \leq T, f_{s}^{y, N}$ is the density of $\mu_{s}^{N}$ with respect to the product measure $\nu_{b\left(\frac{y}{N}\right)}^{\alpha, N}$ with constant profile $b\left(\frac{y}{N}\right)$. The density $\left(\tau_{-\left(y-\ell e_{1}\right)} \bar{f}_{T}^{y, N}\right)^{\ell}$ stands for the conditional expectation of $\tau_{-\left(y-\ell e_{1}\right)} \bar{f}_{T}^{y, N}$ with respect to the $\sigma$-algebra generated by $\left\{\eta(z): z \in \Lambda_{\ell}(0)\right\}$.

Remark that, since the Dirichlet form is convex and since the conditional expectation is an average,

$$
\begin{aligned}
\tilde{D}_{\ell, 0}^{b, \frac{y}{N}}\left(\sqrt{\left(\tau_{-\left(y-\ell e_{1}\right)} \bar{f}_{T}^{y, N}\right)^{\ell}}, \nu_{b(y / N)}^{\alpha, \ell}\right) & \leq \tilde{D}_{\ell, 0}^{b, \frac{y}{N}}\left(\sqrt{\tau_{-\left(y-\ell e_{1}\right)} \bar{f}_{T}^{y, N}}, \nu_{b(y / N)}^{\alpha, N}\right) \\
& =\mathcal{D}_{\ell, y-\ell e_{1}}^{b}\left(\sqrt{\bar{f}_{T}^{y, N}}, \nu_{b(y / N)}^{\alpha, N}\right) \\
& \leq \frac{1}{T} \int_{0}^{T} \mathcal{D}_{\ell, y-\ell e_{1}}^{b}\left(\sqrt{f_{s}^{y, N}}, \nu_{b(y / N)}^{\alpha, N}\right) d s .
\end{aligned}
$$

Applying Lemma 3.9 we obtain from (3.41)

$$
\begin{aligned}
N^{1-d} \sum_{y \in \Gamma_{N}} \tilde{D}_{\ell, 0}^{b, \frac{y}{N}}\left(\sqrt{\left(\tau_{-\left(y-\ell e_{1}\right)} \bar{f}_{T}^{y, N}\right)^{\ell}}, \nu_{b(y / N)}^{\alpha, \ell}\right) & \\
& \leq \frac{1}{T} \int_{0}^{T}\left\{N^{1-d} \sum_{y \in \Gamma_{N}} \mathcal{D}_{\ell, y-\ell e_{1}}^{b}\left(\sqrt{f_{s}^{y, N}}, \nu_{b(y / N)}^{\alpha, N}\right)\right\} d s \\
& \leq 2 \frac{1}{T} \int_{0}^{T}\left\{N^{1-d} \sum_{y \in \Gamma_{N}} \mathcal{D}_{\ell, y-\ell e_{1}}^{b}\left(\sqrt{h_{s}^{N}}, \nu_{\gamma(\cdot)}^{\alpha, N}\right)\right\} d s+C_{0} \frac{\ell^{d+1}}{N^{2}} \\
& \leq \frac{C_{T}}{N}+C_{0} \frac{\ell^{d+1}}{N^{2}},
\end{aligned}
$$

for some constant $C_{T}$ that depends on $T$. By the same argument we obtain the bound on the Dirichlet form $\mathcal{D}_{\ell, 0}^{0}$,

$$
N^{1-d} \sum_{y \in \Gamma_{N}} \mathcal{D}_{\ell, 0}^{0}\left(\sqrt{\left(\tau_{-\left(y-\ell e_{1}\right)} \bar{f}_{T}^{y, N}\right)^{\ell}}, \nu_{b(y / N)}^{\alpha, \ell}\right) \leq \frac{C_{T}}{N}+C_{0} \frac{\ell^{d}}{N^{2}} .
$$

For $N$ fixed and large enough, there exists a constant $C_{T}$, such that for all positive integer $k \geq 1$, applying (3.42) and (3.43), we can bound by above the expectation (3.38) as following

$$
\begin{array}{r}
T\|G\|_{\infty} N^{1-d} \sum_{y \in \Gamma_{N}^{+}}\left\{\int\left|\eta^{\ell}(0)-b(y / N)\right|\left(\tau_{-\left(y-\ell e_{1}\right)} \bar{f}_{T}^{y, N}\right)^{\ell} d \nu_{b(y / N)}^{\alpha, \ell}(\eta)-k \mathcal{D}_{\ell, 0}^{0}\left(\sqrt{\left.\left(\tau_{-\left(y-\ell e_{1}\right)} \bar{f}_{T}^{y, N}\right)^{\ell}, \nu_{b(y / N)}^{\alpha, \ell}\right)}\right.\right. \\
\left.-k \tilde{D}_{\ell, 0}^{b, \frac{y}{N}}\left(\sqrt{\left(\tau_{-\left(y-\ell e_{1}\right)} \bar{f}_{T}^{y, N}\right)^{\ell}}, \nu_{b(y / N)}^{\alpha, \ell}\right)\right\}+\frac{k}{N}\left(C_{T}+\frac{\ell^{d}(\ell+1)}{N}\right) .
\end{array}
$$

This last expression is bounded above by

$$
\begin{array}{r}
T\|G\|_{\infty} N^{1-d} \sum_{y \in \Gamma_{N}^{+}} \sup _{f \in \mathcal{A}_{\ell}^{\frac{y}{N}}}\left\{\int\left|\eta^{\ell}(0)-b(y / N)\right| f(\eta) d \nu_{b(y / N)}^{\alpha, \ell}(\eta)-k \mathcal{D}_{\ell, 0}^{0}\left(\sqrt{f}, \nu_{b(y / N)}^{\alpha, \ell}\right)\right. \\
\left.-k \tilde{D}_{\ell, 0}^{b, \frac{y}{N}}\left(\sqrt{f}, \nu_{b(y / N)}^{\alpha, \ell}\right)\right\}+\frac{k}{N}\left(C_{T}+\frac{\ell^{d}(\ell+1)}{N}\right),
\end{array}
$$


where, for $u \in \Gamma$,

$$
\mathcal{A}_{\ell}^{u}=\left\{f: \quad f \geq 0, \quad \int f(\xi) d \nu_{b(u)}^{\alpha, \ell}(\xi)=1\right\}
$$

Further, since the function

$$
u \rightarrow \sup _{f \in \mathcal{A}_{\ell}^{u}}\left\{\int\left|\eta^{\ell}(0)-b(u)\right| f(\eta) d \nu_{b(u)}^{\alpha, \ell}(\eta)-k \mathcal{D}_{\ell, 0}^{0}\left(\sqrt{f}, \nu_{b(u)}^{\alpha, \ell}\right)-k \tilde{D}_{\ell, 0}^{b, u}\left(\sqrt{f}, \nu_{b(u)}^{\alpha, \ell}\right)\right\}
$$

is continuous on $\Gamma$, from Lemma 3.5, for all positive integers $\ell$ and $k$, the limit when $N \uparrow \infty$ of the expression (3.44) is equal to

$$
T\|G\|_{\infty} \int_{\Gamma} d u \operatorname{IE}\left[\sup _{f \in \mathcal{A}_{\ell}^{u}}\left\{\int\left|\eta^{\ell}(0)-b(u)\right| f d \nu_{b(u)}^{\alpha, \ell}(\eta)-k \mathcal{D}_{\ell, 0}^{0}\left(\sqrt{f}, \nu_{b(u)}^{\alpha, \ell}\right)-k \tilde{D}_{\ell, 0}^{b, u}\left(\sqrt{f}, \nu_{b(u)}^{\alpha, \ell}\right)\right\}\right] .
$$

Since $\int\left|\eta_{s}^{\ell}(0)-b(u)\right| f d \nu_{b(u)}^{\alpha, \ell}(\eta) \leq C_{b}$ for some positive constant $C_{b}$ that depends on $\|b\|_{\infty}$, the integral on $\Gamma$ in the last expression is bounded by

$$
\int_{\Gamma} d u \mathbb{E}\left[\sup _{f \in \mathcal{A}_{\ell, k, C_{b}}^{u}}\left\{\int\left|\eta^{\ell}(0)-b(u)\right| f(\eta) d \nu_{b(u)}^{\alpha, \ell}(\eta)\right\}\right],
$$

where for a positive constant $C, \mathcal{A}_{\ell, k, C}^{u}$ is the following set of densities,

$$
\mathcal{A}_{\ell, k, C}^{u}=\left\{f \in \mathcal{A}_{\ell}^{u}, \quad \tilde{D}_{\ell, 0}^{b, u}\left(\sqrt{f}, \nu_{b(u)}^{\alpha, \ell}\right) \leq \frac{C}{k}, \quad \mathcal{D}_{\ell, 0}^{0}\left(\sqrt{f}, \nu_{b(u)}^{\alpha, \ell}\right) \leq \frac{C}{k}\right\} .
$$

We first consider the limit when $k \uparrow \infty$ and use the usual technics in the replacement lemma. Since for any $\ell>1$, any constant $C>0$ and any $u \in \Gamma$ the sets $\mathcal{A}_{\ell, k, C}^{u}$ are compacts for the weak topology, for all $\ell>1$

$$
\limsup _{k \rightarrow \infty} \sup _{f \in \mathcal{A}_{\ell, k, C}^{u}}\left\{\int\left|\eta^{\ell}(0)-b(u)\right| f(\eta) d \nu_{b(u)}^{\alpha, \ell}(\eta)\right\}=\sup _{f \in \mathcal{A}_{\ell, C}^{u}}\left\{\int\left|\eta^{\ell}(0)-b(u)\right| f(\eta) d \nu_{b(u)}^{\alpha, \ell}(\eta)\right\}
$$

where

$$
\mathcal{A}_{\ell, C}^{u}=\left\{f \in \mathcal{A}_{\ell}^{u}, \quad \tilde{D}_{\ell, 0}^{b, u}\left(\sqrt{f}, \nu_{b(u)}^{\alpha, \ell}\right)=0, \quad \mathcal{D}_{\ell, 0}^{0}\left(\sqrt{f}, \nu_{b(u)}^{\alpha, \ell}\right)=0\right\} .
$$

By dominated convergence theorem, it is then enough to show that,

$$
\limsup _{\ell \rightarrow \infty} \mathbb{E}\left[\sup _{f \in \mathcal{A}_{\ell, C}^{u}}\left\{\int\left|\eta^{\ell}(0)-b(u)\right| f(\eta) d \nu_{b(u)}^{\alpha, \ell}(\eta)\right\}\right]=0 .
$$

Now, it is easy to see that, due to the presence of the jumps of particles in the Dirichlet form $\mathcal{D}_{\ell, 0}^{0}$ and the presence of the creation and destruction of particles in $\tilde{D}_{\ell, 0}^{b, u}$ the set $\mathcal{A}_{\ell, C}^{u}=\{1\}$. Thus, to conclude the proof of the lemma, it remains to apply the usual law of large numbers.

Proof of Proposition 3.4 Let $Q^{*}$ be a limit point of the sequence $\left(Q_{\mu^{N}}\right)_{N \geq 1}$ and let $\left(Q_{\mu^{N} k}\right)_{k \geq 1}$ be a subsequence converging to $Q^{*}$. By Lemma $3.2 Q^{*}$ is concentrated on the trajectories that are in $L^{2}\left([0, T] ; H^{1}(\Lambda)\right)$. For $0<c<1$ and for $\mu(\cdot, \cdot) \in D\left([0, T], \mathcal{M}_{1}^{0}(\Lambda)\right)$, such that $\mu(t, d u)=\rho(t, u) d u$ with $\rho(\cdot, \cdot) \in L^{2}\left([0, T] ; H^{1}(\Lambda)\right)$, denote by $F_{c}^{G}(\mu)$ the functional

$$
\begin{aligned}
F_{c}^{G}(\mu(\cdot, \cdot))= & \int_{0}^{T} d s \int_{\Lambda_{(1-c)}} d u\left\{G_{s}(u)(2 c)^{-1}\left[\rho\left(s, u+c e_{1}\right)-\rho\left(s, u-c e_{1}\right)\right]\right\} \\
& +\int_{0}^{T} d s \int_{\Lambda} d u \partial_{e_{1}} G_{s}(u) \rho(s, u)-\int_{0}^{T} d s\left\{\int_{\Gamma} b(u) \mathbf{n}_{1}(u) G_{s}(u) \mathrm{d} S\right\}
\end{aligned}
$$


From Lemma 3.11 and the continuity of the function $\mu \rightarrow \hat{F}_{a, c}^{G}(\mu)$, we have

$$
\limsup _{c \rightarrow 0} E^{Q^{*}}\left[\left|F_{c}^{G}(\mu)\right|\right]=0 \text {. }
$$

On the other hand, an integration by parts and Taylor expansion up to the second order of the function $G_{s}(\cdot)$ permit to rewrite $F_{c}^{G}$ as

$$
\begin{aligned}
F_{c}^{G}(\mu(\cdot, \cdot))= & \int_{0}^{T} \frac{1}{2 c} \int_{\left(\Lambda \backslash \Lambda_{(1-2 c)}\right)^{+}} G_{s}(u) \rho(s, u) d u d s-\int_{0}^{T} \frac{1}{2 c} \int_{\left(\Lambda \backslash \Lambda_{(1-2 c)}\right)^{-}} G_{s}(u) \rho(s, u) d u d s \\
& -\int_{0}^{T} d s \int_{\Gamma} b(u) \mathbf{n}_{1}(u) G_{s}(u) \mathrm{dS}+R(c),
\end{aligned}
$$

where $R(c) \equiv R(G, c)$ is a function vanishing as $c \downarrow 0$. Further one has, see Theorem 5.3.2. of [EG], that

$$
\lim _{r \rightarrow 0} \frac{1}{|B(u, r) \cap \Lambda|} \int_{B(u, r) \cap \Lambda} \rho(s, y) d y=\operatorname{Tr}(\rho(s, u)) \quad \text { a.e } \quad u \in \Gamma, \forall s,
$$

and then by dominated convergence theorem

$$
\lim _{c \rightarrow 0} F_{c}^{G}(\mu(\cdot, \cdot))=\int_{0}^{T} d s \int_{\Gamma}(\operatorname{Tr}(\rho(s, u))-b(u)) \mathbf{n}_{1}(u) G_{s}(u) \mathrm{dS} .
$$

This together with (3.45) implies

$$
E^{Q^{*}}\left[\left|\int_{0}^{T} d s \int_{\Gamma}(\operatorname{Tr}(\rho(s, u))-b(u)) \mathbf{n}_{1}(u) G_{s}(u) \mathrm{dS}\right|\right]=0
$$

which concludes the proof.

\section{Proof of Theorem 2.2}

The main problem in proving Theorem 2.2 is that we cannot associate to the stationary measure $\nu_{s}{ }^{\alpha, N}$ a macroscopic profile according to definition (2.26). If this would be the case the result would be a corollary of Theorem 2.1. Denote by $\mathbf{Q}^{N}:=Q_{\nu_{s}{ }^{\alpha, N}}^{N, \alpha}$ the probability measure on the Skorohod space $D([0, T], \mathcal{M})$ induced by the Markov process $\left(\pi_{t}^{N}\right) \equiv\left(\pi_{N}\left(\eta_{t}\right)\right)$, when the initial measure is $\nu_{s}{ }^{\alpha, N}$. Denote by $\mathcal{A}_{T} \subset$ $D([0, T], \mathcal{M})$ the class of profiles $\rho(\cdot, \cdot)$ that satisfies conditions (IB1), (IB2) and (IB3). The first step to show Theorem 2.2 consists in proving that all limit points of the sequence $\left(\mathbf{Q}^{N}\right)$ are concentrated on $\mathcal{A}_{T}$ :

Proposition 4.1 The sequence of probability measures $\left(\mathbf{Q}^{N}\right)$ is weakly relatively compact and all its converging subsequences converge to the some limit $\mathbf{Q}^{*}$ that is concentrated on the absolutely continuous measures $\pi(t, d u)=\rho(t, u) d u$ whose density $\rho$ satisfying (IB1), (IB2) and (IB3).

The proof of Proposition 4.1 follows the same steps needed to show Theorem 2.1. We just have to show the analogous of Lemmas 3.6, 3.7, 3.8 and 3.9 when the measure $\mu^{N}$ in the statements of these lemmas is replaced by $\nu_{s}{ }^{\alpha, N}$. The only lemma to be slightly modified is Lemma 3.8, see Lemma 4.2 given next. Recall that $\gamma: \Lambda \rightarrow(0,1)$ is a smooth profile equal to $b$ at the boundary of $\Lambda$. Let $h^{N}$ be the density of $\nu_{s}{ }^{\alpha, N}$ with respect to the measure $\nu_{\gamma(\cdot)}^{\alpha, N}$. 
Lemma 4.2 There exists positive constant $C=C\left(\|\nabla \gamma\|_{\infty}\right)$ depending only on $\gamma(\cdot)$ such that for any a $>0$

$$
(1-a) \mathcal{D}_{N}^{0}\left(\sqrt{h^{N}}, \nu_{\gamma(\cdot)}^{\alpha, N}\right)+\mathcal{D}_{N}^{b}\left(\sqrt{h^{N}}, \nu_{\gamma(\cdot)}^{\alpha, N}\right) \leq \frac{C}{a} N^{d-2}
$$

Proof. By the stationary of $\nu_{s}^{\alpha, N}$,

$$
\partial_{t} H_{N}(t)=\int_{\mathcal{S}_{N}} h^{N} \mathcal{L}_{N} \log \left(h^{N}\right) d \nu_{\gamma(\cdot)}^{\alpha, N}=0 .
$$

Recalling that the generator $\mathcal{L}_{N}$ has two pieces and applying the basic inequality $a(\log b-\log a) \leq-(\sqrt{a}-$ $\sqrt{b})^{2}+(b-a)$ for positive $a$ and $b$, we obtain

$$
\begin{aligned}
0=\int_{\mathcal{S}_{N}} h^{N} \mathcal{L}_{N} \log \left(h^{N}\right) d \nu_{\gamma(\cdot)}^{\alpha, N} \leq & -2 N^{2} \mathcal{D}_{N}^{0}\left(\sqrt{h^{N}}, \nu_{\gamma(\cdot)}^{\alpha, N}\right)-2 N^{2} \mathcal{D}_{N}^{b}\left(\sqrt{h^{N}}, \nu_{\gamma(\cdot)}^{\alpha, N}\right) \\
& +N^{2} \int_{\mathcal{S}_{N}} \mathcal{L}_{N}^{0} h^{N} d \nu_{\gamma(\cdot)}^{\alpha, N}+N^{2} \int_{\mathcal{S}_{N}} \mathcal{L}_{N}^{b} h^{N} d \nu_{\gamma(\cdot)}^{\alpha, N}
\end{aligned}
$$

We then apply the same computation as in the proof of Lemma 3.8, ((3.15) and (3.16)).

\section{Proof of Theorem 2.2}

Let $\mathbf{Q}^{*}$ be a limit point of $\left(\mathbf{Q}^{N}\right)$ and $\left(\mathbf{Q}^{N_{k}}\right)$ be a sub-sequence converging to $\mathbf{Q}^{*}$. Let $\bar{\rho}$ be the stationary solution of (2.15), see (2.24). We have by Proposition 4.1 the following:

$$
\begin{aligned}
\lim _{k \rightarrow \infty} q_{N_{k}}(\alpha) & :=\lim _{k \rightarrow \infty} \mathbf{Q}^{N_{k}}\left\{\left|\left\langle\pi_{T}^{N}, G\right\rangle-\langle\bar{\rho}(u) d u, G\rangle\right|\right\} \\
& =\mathbf{Q}^{*}\left\{|\langle\rho(T, \cdot), G\rangle-\langle\bar{\rho}(u) d u, G\rangle| \mathbb{I}\left\{\mathcal{A}_{T}\right\}(\rho)\right\} \\
& \left.\leq\|G\|_{\infty} \mathbf{Q}^{*}\left\{\|\rho(T, \cdot)-\bar{\rho}(\cdot)\|_{1} \mathbb{I}\left\{\mathcal{A}_{T}\right\}(\rho)\right\}\right\} .
\end{aligned}
$$

Denote by $\rho^{0}(\cdot, \cdot)\left(\right.$ resp. $\left.\rho^{1}(\cdot, \cdot)\right)$ the element of $\mathcal{A}_{T}$ with initial condition $\rho^{0}(0, \cdot) \equiv 0\left(\right.$ resp. $\left.\rho^{1}(0, \cdot) \equiv 1\right)$. From Lemma 5.8, each profile $\rho(\cdot, \cdot) \in \mathcal{A}_{T}$ is such that for all $t \geq 0, \lambda\left\{u \in \Lambda: 0 \leq \rho^{0}(t, u) \leq \rho(t, u) \leq\right.$ $\left.\rho^{1}(t, u) \leq 1\right\}=1$ and $\lambda\left\{u \in \Lambda: \rho^{0}(t, u) \leq \bar{\rho}(u) \leq \rho^{1}(t, u)\right\}=1$, where $\lambda$ is the Lebesgue measure on $\Lambda$. Therefore

$$
\lim _{k \rightarrow \infty} q_{N_{k}}(\alpha) \leq\|G\|_{\infty}\left\|\rho^{0}(T, \cdot)-\rho^{1}(T, \cdot)\right\|_{1}, \quad I P=1 .
$$

To conclude the proof, it is enough to let $T \uparrow \propto$ and to apply Theorem 5.1. 


\section{Appendix}

In this section we show the global stability of the stationary solution of (2.15).

Theorem 5.1 Global stability. Let $D(\cdot)$ be Lipschitz. Let $\rho\left(t, \rho_{0}\right)$ be the solution of (2.15) with initial datum $\rho_{0}, 0 \leq \rho_{0}(u) \leq 1, u \in \Lambda$, and $\bar{\rho}$ the stationary solution of (2.15). We have

$$
\lim _{t \rightarrow \infty} \int_{\Lambda}|\rho(t, u)-\bar{\rho}(u)|^{p} d u=0
$$

for all $p \geq 1$.

The proof of the theorem is based on an extensive use of monotone methods, see [S]. We were not able to find the precise reference, so we briefly sketch it for completeness. We need to introduce some extra notation. Let $\mathcal{C}^{1,2}([0, T] \times \Lambda)$ be the space of functions from $[0, T] \times \Lambda$ to $\mathbb{R}$ twice continuously differentiable in $\Lambda$ with continuous time derivative. Denote by

$$
\mathcal{G}:=\left\{G \in \mathcal{C}^{1,2}([0, T] \times \Lambda), G(t, u)=G_{t}(u) \quad \text { pointwise positive, } \quad G(t, u)=0, \forall u \in \Gamma, \forall t \in[0, T]\right\}
$$

It is convenient to reformulate the notion of weak solution of $(2.15)$ as following. A function $\rho(\cdot, \cdot):[0, T] \times$ $\Lambda \rightarrow[0,1]$ is a weak solution of the initial-boundary value problem $(2.15)$ if $\rho \in L^{2}\left(0, T ; H^{1}(\Lambda)\right)$ and for every $G \in \mathcal{G}$

$$
\begin{aligned}
& \int_{\Lambda} d u\left\{G_{T}(u) \rho(T, u)-G_{0}(u) \rho_{0}(u)\right\}-\int_{0}^{T} d s \int_{\Lambda} d u\left(\partial_{s} G_{s}\right)(u) \rho(s, u) \\
& =\sum_{i, j} \int_{0}^{T} d s\left\{\int_{\Lambda} d u A_{i, j}(\rho(s, u)) \frac{\partial^{2}}{\partial_{i, j}} G_{s}(u)-\int_{\Gamma} A_{i, j}(b(u)) \partial_{n_{1}} G(s, u) d S\right\}
\end{aligned}
$$

where $A_{i, j}(\rho)=\int_{0}^{\rho} D_{i, j}\left(\rho^{\prime}\right) d \rho^{\prime}$. A function $\rho^{+}(\cdot, \cdot):[0, T] \times \Lambda \rightarrow \mathbb{R}$ is a weak upper solution of the initial-boundary value problem $(2.15)$ if $\rho^{+} \in L^{2}\left(0, T ; H^{1}(\Lambda)\right)$ and for all $G \in \mathcal{G}$ we have

$$
\left\{\begin{array}{l}
\sum_{i, j} \int_{0}^{T} d s\left\{\int_{\Lambda} d u A_{i, j}\left(\rho^{+}(s, u)\right) \frac{\partial^{2}}{\partial_{i, j}} G_{s}(u)-\int_{\Gamma} A_{i, j}\left(\rho^{+}(s, u)\right) \partial_{n_{1}} G(s, u) d S\right\} \\
-\int_{\Lambda} d u\left\{G_{T}(u) \rho^{+}(T, u)-G_{0}(u) \rho_{0}^{+}(u)\right\}-\int_{0}^{T} d s \int_{\Lambda} d u\left(\partial_{s} G_{s}\right)(u) \rho^{+}(s, u) \leq 0 \\
\operatorname{Tr}\left(\rho^{+}(t, \cdot)\right) \geq b(\cdot) \quad \text { on } \quad \Gamma \\
\rho^{+}(0, u) \geq \rho_{0}(u) \quad u \in \Lambda
\end{array}\right.
$$

A weak lower solution $\rho^{-}(\cdot, \cdot):[0, T] \times \Lambda \rightarrow \mathbb{R}$ is defined reversing the inequality in (5.2).

By a solution of the stationary problem (2.15) we mean a function $\bar{\rho} \in H^{1}(\Lambda)$ so that for all $G \in \mathcal{C}^{2}(\Lambda)$, pointwise positive vanishing on $\Gamma$

$$
\left.\sum_{i, j}\left\{\int_{\Lambda} d u A_{i, j}(\bar{\rho}(u))\right) \frac{\partial^{2}}{\partial_{i, j}} G(u)-\int_{\Gamma} A_{i, j}(b(u)) \partial_{n_{1}} G(u) d S\right\}=0
$$


As before we define upper and lower solutions of the stationary problem (5.3). A function $\bar{\rho}^{+}$is an upper solution for the stationary problem (5.3) if $\bar{\rho}^{+} \in H^{1}(\Lambda)$ and for all $G \in \mathcal{C}^{2}(\Lambda)$, pointwise positive vanishing on $\Gamma$,

$$
\left\{\begin{array}{l}
\left.\sum_{i, j}\left\{\int_{\Lambda} d u A_{i, j}\left(\bar{\rho}^{+}(u)\right)\right) \frac{\partial^{2}}{\partial_{i, j}} G(u)-\int_{\Gamma} A_{i, j}\left(\bar{\rho}^{+}(u)\right) \partial_{n_{1}} G(u) d S\right\} \leq 0, \\
\operatorname{Tr}\left(\bar{\rho}^{+}\right) \geq b \quad \text { on } \quad \Gamma
\end{array}\right.
$$

A lower solution of the stationary problem (5.3) is defined reversing the inequality in (5.4).

To apply the monotone method we first show the following comparison principle.

Lemma 5.2 Let $\rho^{1}$ (resp. $\rho^{2}$ ) be a lower solution (resp. upper solution) of (2.15), $\partial_{t} \rho^{i} \in L^{2}\left(0, T ; H^{-1}(\Lambda)\right)$, for $i=1,2$. If there exists $s \geq 0$ such that

$$
\lambda\left\{u \in \Lambda: \rho^{1}(s, u) \leq \rho^{2}(s, u)\right\}=1,
$$

where $\lambda$ is the Lebesgue measure on $\Lambda$, then for all $t \geq s$

$$
\lambda\left\{u \in \Lambda: \quad \rho^{1}(t, u) \leq \rho^{2}(t, u)\right\}=1 .
$$

Proof Take $s<t<T$ and $\delta>0$. Denote by $F_{\delta}$ the function defined by

$$
F_{\delta}(a):=\frac{a^{2}}{2 \delta} \mathbb{I}_{\{0 \leq a \leq \delta\}}+(a-\delta / 2) \mathbb{I}_{\{a>\delta\}}, \quad a \in \mathbb{R} .
$$

Let $A_{\delta}:=A_{\delta}(T)$ be the set

$$
A_{\delta}=\left\{(t, u) \in[0, T] \times \Lambda: 0 \leq \rho^{1}(t, u)-\rho^{2}(t, u) \leq \delta\right\} .
$$

By definition $\operatorname{Tr}\left(\rho^{1}-\rho^{2}\right) \leq 0$ a.e. and therefore $\operatorname{Tr}\left(F_{\delta}^{\prime}\left(\rho^{1}-\rho^{2}\right)\right)=0$. Since $\rho^{1}\left(\rho^{2}\right)$ is lower (upper) solution of (2.15), we have that

$$
\begin{aligned}
\int_{s}^{t} d \tau \frac{\partial}{\partial \tau} \int_{\Lambda} F_{\delta}\left(\rho^{1}(\tau, u)-\rho^{2}(\tau, u)\right)=\int_{\Lambda} d u & F_{\delta}\left(\rho^{1}(t, u)-\rho^{2}(t, u)\right)-\int_{\Lambda} d u F_{\delta}\left(\rho^{1}(s, u)-\rho^{2}(s, u)\right) \\
\leq & -\delta^{-1} \int_{s}^{t} d \tau \int_{A_{\delta}} d u \nabla\left(\rho^{1}-\rho^{2}\right) \cdot\left\{D\left(\rho^{1}\right) \nabla \rho^{1}-D\left(\rho^{2}\right) \nabla \rho^{2}\right\} \\
= & -\delta^{-1} \int_{s}^{t} d \tau \int_{A_{\delta}} d u \nabla\left(\rho^{1}-\rho^{2}\right) \cdot D\left(\rho^{1}\right) \nabla\left(\rho^{1}-\rho^{2}\right) \\
& -\delta^{-1} \int_{s}^{t} d \tau \int_{A_{\delta}} d u \nabla\left(\rho^{1}-\rho^{2}\right) \cdot\left\{D\left(\rho^{1}\right)-D\left(\rho^{2}\right)\right\} \nabla \rho^{2} .
\end{aligned}
$$

Since $D(\cdot)$ is strictly positive, see (2.19), the third line of (5.5) can be estimated by above

$$
-\frac{1}{\delta} \int_{s}^{t} d \tau \int_{A_{\delta}} d u \nabla\left(\rho^{1}-\rho^{2}\right) \cdot D\left(\rho^{1}\right) \nabla\left(\rho^{1}-\rho^{2}\right) \leq-\frac{1}{\delta C} \int_{s}^{t} d \tau \int_{A_{\delta}} d u\left\|\nabla\left(\rho^{1}-\rho^{2}\right)\right\|^{2} .
$$


Further, by the Lipschitz property of $D(\cdot)$ we have on the set $A_{\delta}, \sup _{1 \leq i, j \leq d}\left|D_{i, j}\left(\rho^{1}\right)-D_{i, j}\left(\rho^{2}\right)\right| \leq M \mid \rho^{1}-$ $\rho^{2} \mid \leq M \delta$ for some positive constant $M$. By Schartz inequality, the last line of (5.5) is bounded by

$$
\delta^{-1} M A \int_{s}^{t} d \tau \int_{A_{\delta}} d u\left\|\nabla\left(\rho^{1}-\rho^{2}\right)\right\|^{2}+\delta M A^{-1} \int_{s}^{t} d \tau \int_{A_{\delta}} d u\left\|\nabla \rho^{2}\right\|^{2}
$$

for every $A>0$. By (5.5), (5.6), (5.7) and choosing $A=M^{-1} C^{-1}$ to cancel the term in (5.6) and the first term of (5.7) we have

$$
\begin{gathered}
\int_{\Lambda} d u F_{\delta}\left(\rho^{1}(t, u)-\rho^{2}(t, u)\right)-\int_{\Lambda} d u F_{\delta}\left(\rho^{1}(s, u)-\rho^{2}(s, u)\right) \\
\leq \delta C^{-1} M^{2} \int_{0}^{T} d \tau \int d u\left\|\nabla \rho^{2}\right\|^{2} .
\end{gathered}
$$

Letting $\delta \downarrow 0$, we conclude the proof of the lemma because $F_{\delta}(\cdot)$ converges to the function $F(a)=a \mathbb{1}_{a \geq 0}$ as $\delta \downarrow 0$.

By Lemma 5.2 we immediately obtain the following corollaries.

Corollary 5.3 Let $m_{0}: \Lambda \rightarrow[0,1]$ be a measurable function. There is a unique weak solution $\rho\left(t, m_{0}\right)$ of the equation (2.15) with initial datum $m_{0}$.

Corollary 5.4 Let $m_{0}$ be a lower stationary solution of (5.3). Let $\rho\left(t, m_{0}\right)$ be the solution of (5.1) with initial datum $m_{0}$ then $\rho(t, u) \geq m_{0}(u)$ a.e in $(u, t)$.

The proof is an immediate consequence of Lemma 5.2 with $\rho^{1}:=m_{0}$ and $\rho^{2}:=\rho$. When the initial datum of solution of (5.8) is an upper stationary solution we have:

Corollary 5.5 Let $m_{1}$ be a upper stationary solution of (5.3). Let $\rho\left(t, m_{1}\right)$ be the solution of (5.1) with initial datum $m_{1}$ then $\rho(t, u) \leq m_{1}(u)$ for $t \in[0, T]$ and $u \in \Lambda$.

Next we show that when a lower (upper) stationary solution $m_{0}\left(m_{1}\right)$ is taken as initial datum, the corresponding solution $\rho\left(t, m_{0}\right)\left(\rho\left(t, m_{1}\right)\right)$ is monotone nondecreasing (nonincreasing) in time.

Lemma 5.6 Under the assumptions of Corollary $5.4 \rho\left(t, m_{0}\right)$ is a nondecreasing solution of (2.15) for all $t \in[0, T]$.

Proof: Corollary 5.4 implies that $\rho\left(s, m_{0}\right) \geq m_{0}$ for all $s \geq 0$, since $m_{0}$ lower solution. Let $\rho\left(t ; \rho\left(s, m_{0}\right)\right)$ be the solution of (5.1) starting at time $t=0$ from $\rho\left(s, m_{0}\right)$. Then $\rho\left(t ; \rho\left(s, m_{0}\right)\right) \geq \rho\left(t, m_{0}\right)$ since the initial datum $\rho\left(s, m_{0}\right) \geq m_{0}$. But $\rho\left(t ; \rho\left(s, m_{0}\right)\right)=\rho\left(t+s, m_{0}\right)$ by uniqueness of weak solution then $\rho\left(t+s, m_{0}\right) \geq$ $\rho\left(t, m_{0}\right) \geq m_{0}$.

Lemma 5.7 Under the assumptions of Corollary $5.5 \rho\left(t, m_{1}\right)$ is a nonincreasing solution of (2.15) for $t \in[0, T]$.

The proof is similar to the one of Lemma 5.6.

Lemma 5.8 Let $m_{0}$ be a lower solution and $m_{1}$ be an upper solution of $(5.3), m_{0}(\cdot) \leq m_{1}(\cdot)$ a.e in $\Lambda$, we have

$$
m_{0} \leq \rho\left(t ; m_{0}\right) \leq \rho\left(t ; m_{1}\right) \leq m_{1} \quad \forall t \in(0, \infty)
$$

29/october/2018; 13:48 
The proof is an immediate consequence of the previous results.

Lemma 5.9 Under the assumption of Lemma 5.8 the solutions $\rho\left(t ; m_{0}\right)$ and $\rho\left(t ; m_{1}\right)$ exist for all $t \in[0, \infty)$ and they converge in $L^{p}(\Lambda)$ for $p \in[1, \infty)$ to limits $\rho_{\star}(\cdot)$ and $\rho^{\star}(\cdot)$, both solutions of (5.3). Further

$$
\rho_{\star}(u) \leq \rho^{\star}(u) \text { a.e. }
$$

Proof: Since $\rho\left(t ; m_{0}\right)$ is nondecreasing in $t$ and $\rho\left(t ; m_{0}\right) \leq m_{1}$ for any $t \geq 0, \rho\left(t ; m_{0}\right)$ converges almost everywhere in $\Lambda$ as $t \rightarrow \infty$ and $\rho_{\star}(\cdot) \in L^{\infty}(\Lambda)$. By the monotone convergence theorem $\rho\left(t ; m_{0}\right) \rightarrow \rho_{\star}(\cdot)$ for $p \in[1, \infty)$. Next we show that $\rho_{\star}(\cdot)$ solves (5.3). Take as test function in (5.1) the following function

$$
\beta(t) F(u) ; \quad F(u)>0 ; \quad C \geq \beta(t)>\delta>0 ; \quad \beta^{\prime}(t) \geq 0,(u, t) \in \Lambda \times \mathbb{R}^{+}
$$

$\beta \in C^{2}\left(R^{+}\right), F \in C^{2}(\Lambda)$ vanishing at the boundary. Then for all $t>0$, see (5.1), we have

$$
\begin{aligned}
& \int_{\Lambda} d u\left\{\beta(t) F(u) \rho(t, u)-\beta(0) F(u) \rho_{0}(u)\right\}-\int_{0}^{t} d s \beta^{\prime}(s) \int_{\Lambda} d u F(u) \rho(s, u) \\
& =\sum_{i, j} \int_{0}^{t} d s \beta(s)\left\{\int_{\Lambda} d u A_{i, j}(\rho(s, u)) \frac{\partial^{2}}{\partial_{i, j}} F(u)-\int_{\Gamma} A_{i, j}(b(u)) \partial_{n_{1}} F(u) d S\right\} .
\end{aligned}
$$

Divide by $t$ the left and right side of (5.9) and then let $t \rightarrow \infty$. For the left side we have

$$
\frac{1}{t}\left\{\int_{\Lambda} d u\left\{\beta(t) F(u) \rho(t, u)-\beta(0) F(u) \rho_{0}(u)\right\}-\int_{0}^{t} d s \beta^{\prime}(s) \int_{\Lambda} d u F(u) \rho(s, u)\right\} \rightarrow 0 .
$$

By continuity of $A(\cdot)$ and since by assumption $\lim _{s \rightarrow \infty} \beta(s)=\beta(\infty)>0$

$$
\begin{aligned}
& \lim _{t \rightarrow \infty} \frac{1}{t} \sum_{i, j} \int_{0}^{t} d s \beta(s)\left\{\int_{\Lambda} d u A_{i, j}(\rho(s, u)) \frac{\partial^{2}}{\partial_{i, j}} F(u)-\int_{\Gamma} A_{i, j}(b(u)) \partial_{n_{1}} F(u) d S\right\} \\
& =\beta(\infty) \sum_{i, j}\left\{\int_{\Lambda} d u A_{i, j}\left(\rho_{\star}(u)\right) \frac{\partial^{2}}{\partial_{i, j}} F(u)-\int_{\Gamma} A_{i, j}(b(u)) \partial_{n_{1}} F(u) d S\right\} .
\end{aligned}
$$

By (5.10) we then obtain

$$
\beta(\infty) \sum_{i, j}\left\{\int_{\Lambda} d u A_{i, j}\left(\rho_{\star}(u)\right) \frac{\partial^{2}}{\partial_{i, j}} F(u)-\int_{\Gamma} A_{i, j}(b(u)) \partial_{n_{1}} F(u) d S\right\}=0 .
$$

Therefore $\rho_{\star}$ is a solution of (5.3). The same can be argued for $\rho^{*}$.

The proof of Theorem 5.1 is a simple consequence of Lemma 5.9 and the unicity of the stationary solution $\rho^{*}=\rho_{\star}$ of $(2.15)$.

Acknowledgements Enza Orlandi is indebted to Assunta Pozio (La Sapienza, Roma) for helpful suggestions about Section 5. She further thanks the University of Rouen and the Institut Henri Poincaré - Centre Emile Borel, (workshop Mécanique statistique, probabilités et systèmes de particules 2008) where part of the work has been done. Mustapha Mourragui thanks J. S. Farfan Vargas for helpful discussions concerning the hydrostatic. We thank Claudio Landim for suggesting the approach to prove hydrostatic 


\section{References}

[BSGJL] L. Bertini, A. De Sole, D. Gabrielli, G. Jona-Lasinio, C. Landim, Large deviation approach to non equilibrium processes in stochastic lattice gases. Bull. Braz. Math. Soc. (N.S.) 37 611-643 (2006).

[BSGJL] L. Bertini, A. De Sole, D. Gabrielli, G. Jona-Lasinio, C. Landim, Large deviations of the empirical current in interacting particle systems. Teor. Veroyatn. Primen. 51 (2006), no. 1, 144-170; translation in Theory Probab. Appl. 51 (2007), no. 1, 2-27

[DL] R. Dautray and J.L. Lions, Analyse mathématique et calcul numérique pour le sciences et les techniques. tome 3, Masson, Paris (1985)

[DFIP] A. De Masi, P. Ferrari, N. Ianiro, E.Presutti, Small deviations from local equilibrium for a process which exhibits hydrodynamical behaviour. II. J. Stat. Phys. 29, 81-93 (1982)

[DEL] B. Derrida, C. Enaud, J.L. Lebowitz, The asymmetric exclusion process and Brownian excursions , J. Stat. Phys. 115, 365-383 (2004)

[DELO] B. Derrida, C. Enaud, C. Landim, S. Olla, Fluctuaction in the weakly asymmetric exclusion process with open boundary conditions. J. Stat. Phys. 121, 271-289, (2005)

[E] L.C. Evans, Partial Differential Equation, American Mathematical Society, (1998).

[EG] L.C. Evans and R. F. Gariepy, Measure Theory and Fine Properties of Functions, Studies in Advanced Mathematics, (1992).

[ELS1] G. Eyink, J. L. Lebowitz and H. Spohn, Hydrodynamics of Stationary Nonequilibrium States for Some Lattice Gas Models. Commun. Math. Phys. 132, 252-283 (1990).

[ELS2] G. Eyink, J. L. Lebowitz and H. Spohn, Lattice Gas Models in contact with Stochastic Reservoirs: Local Equilibrium and Relaxation to the Steady State. Commun. Math. Phys. 140, 119-131 (1991).

[FM] A. Faggionato and F. Martinelli, Hydrodynamic limit of a disordered lattice gas. Prob. Th. Rel. Fields 127, (4), (2003), 535-608.

[FLM] J. S. Farfan Vargas, C. Landim and M. Mourragui, Hydrodynamic behavior and large deviations of boundary driven exclusion processes in dimension $d>1$. in preparation

[GPV] M.Z. Guo, G. Papanicolau and S.R.S.Varadhan, Nonlinear diffusion limit for a system with nearest neighbor interactions. Comm. Math. phys. 118, 31-59, (1988).

[KL] C. Kipnis and C. Landim, Hydrodynamic limit of interacting particle systems. Springer-Verlag, (1999).

[LSU] O. A. Ladyzenskaja, V.A. Solonnikov, N.N. Uralceva, Linear and quasi linear equations of parabolic type. AMS. 23, (1968). 1998.

[KLO] C. Kipnis, C. Landim and S.Olla, Macroscopic properties of a stationary nonequilibrium distribution for a non-gradient interacting particle system. Ann. Inst. H. Poincaré. 31, 191-221, (1995).

[KW] K.W. Kehr, T. Wichman, Diffusion Processes: experiment, theory of simulations . Lectures Notes In Physics 438, (1994)

[Li] T. Liggett, Interacting particles systems. (1985), Springer, Berlin.

[LMS] C. Landim, M. Mourragui and S. Sellami, Hydrodynamical limit for a nongradient interacting particle system with stochastic reservoirs. Probab. Theory and Appl. 45, N. 4, 2000.

[S] D. H. Sattinger, Monotone methods in Nonlinear Elliptic and Parabolic Boundary Value Problems.. Indiana University Math. J. . 21, N. 11, 1972.

[Sp] H. Spohn, Large scale dynamics of interacting particles. (1991), Springer, Berlin. 
[Sp1] H. Spohn, Long range correlations for stochastic lattice gases in a non-equilibrium steady state. J. Phys. A:Math. Gen. 16 4275-4291, 1983 